\title{
Phenomenology of 2HDM with vectorlike quarks
}

\author{
A. Arhrib, ${ }^{1,2, *}$ R. Benbrik, ${ }^{3, \dagger}$ S. J. D. King, ${ }^{4, \$}$ B. Manaut, ${ }^{5, \S}$ S. Moretti, ${ }^{4, \|}$ and C. S. Un ${ }^{6,7, \pi}$ \\ ${ }^{1}$ Faculté des Sciences et Techniques, Abdelmalek Essaadi University, B.P. 416, Tangier, Morocco \\ ${ }^{2}$ Physics Division, National Center for Theoretical Sciences, Hsinchu, Taiwan \\ ${ }^{3}$ MSISM Team, Faculté Polydisciplinaire de Safi, Sidi Bouzid, BP 4162, Safi, Morocco \\ ${ }^{4}$ School of Physics and Astronomy, University of Southampton, Southampton, SO17 1BJ, United Kingdom \\ ${ }^{5}$ Equipe de Recherche en Physique Théorique et Matériaux (ERPTM), Faculté Polydisciplinaire, \\ Université Sultan Moulay Slimane, Béni Mellal, Morocco \\ ${ }^{6}$ Center for Fundamental Physics, Zewail City of Science and Technology, 6 October City, Giza, Egypt \\ ${ }^{7}$ Department of Physics, Uludağ University, Bursa 16059, Turkey
}

(Received 22 December 2016; revised manuscript received 28 February 2018; published 15 May 2018)

In this paper, we examine the consistency of the Large Hadron Collider (LHC) data collected during Runs 1 and 2 by the ATLAS and CMS experiments with the predictions of a 2-Higgs doublet model embedding vectorlike quarks (VLQs) for $p p \rightarrow H, A$ production and $H, A \rightarrow \gamma \gamma$ decay mechanisms, respectively, of (nearly) degenerate $C P$-even $(H)$ and $C P$-odd $(A)$ Higgs bosons. We show that a scenario containing one single VLQ with electromagnetic charge $2 / 3$ can explain the above ATLAS and CMS data for masses in the region $350 \mathrm{GeV} \leq m_{\mathrm{VLQ}} \leq 1.5 \mathrm{TeV}$ or so, depending on $\tan \beta$, and for several values of the mixing angle between the top quark $(t)$ and its VLQ counterpart $(T)$. We then perform a global fit onto the model by including all relevant experimental as well as theoretical constraints. The surviving samples of our analysis are discussed within $2 \sigma$ of the LHC measurements. Additionally, we also comment on the recent anomalous result reported by CMS using Run 2 data on the associated Standard Model Higgs boson production with top quark pairs $p p \rightarrow t \bar{t} h$ with an observed significance of $3.3 \sigma$. Other than these specific examples, we also present a phenomenological analysis of the main features of the model, including the most promising $T$ decay channels.

DOI: 10.1103/PhysRevD.97.095015

\section{INTRODUCTION}

After the Higgs boson discovery in Run 1 of the Large Hadron Collider (LHC) at CERN [1,2], the ATLAS and CMS collaborations have carried out a broad program looking for new physics beyond the Standard Model (BSM) at the TeV scale. In particular, they have developed a powerful detection machinery of spin-0 resonances. However, new physics phenomena might take different forms from those established so far, and once discovered, they will require a complementary effort in order to understand their underlying nature. In fact, there are

\footnotetext{
*aarhrib@gmail.com

r.benbrik@uca.ac.ma

*sjd.king@soton.ac.uk

§.manaut@usms.ma

"stefano@soton.ac.uk

"csalih@zewailcity.edu.eg
}

Published by the American Physical Society under the terms of the Creative Commons Attribution 4.0 International license. Further distribution of this work must maintain attribution to the author(s) and the published article's title, journal citation, and DOI. Funded by SCOAP ${ }^{3}$. already several potential anomalies in the Run 1 Higgs data indicating possible deviations from the SM expectations in the Higgs sector. In particular, the signal strength of the $t \bar{t} h$ associated production mode is the most prominent one, while milder effects are seen in the fits to those extracted from the other production modes active at the LHC, i.e., gluon-gluon scattering (ggh), Higgs-strahlung $(\mathrm{hV})$, and vector boson fusion (VBF).

A possibility to capture at once all such anomalies is offered by the presence of vectorlike quark (VLQs) as they can, on the one hand, affect the SM-like Higgs (henceforth denoted by $h$ ) production and decay phenomenology (as they would enter the loops mediating the processes $g g \rightarrow h$ as well as $h \rightarrow \gamma \gamma$ and $Z \gamma$ ) and, on the other hand, mediate a $t \bar{t} h$ final state (tth). Intriguingly, the same VLQs affecting $h$ processes would also do so for heavier Higgs states, which may pertain to a BSM scenario. To be specific in defining our framework, we investigate the effects of VLQs in the production and decay of Higgs bosons within a 2-Higgs doublet model type-II (2HDM-II). In practice, we concentrate here on new states of matter that are heavy spin $1 / 2$ particles that transform as triplets under color but, unlike SM quarks, their left- and right-handed couplings have the same electroweak (EW) quantum numbers. Furthermore, 
their couplings to Higgs bosons do not participate in the standard EW symmetry breaking (EWSB) dynamics onset by the Higgs mechanism; hence they are not of Yukawa type (i.e., proportional to the mass), rather they are additional parameters, which can then be set as needed in order to achieve both compliance with present data and predict testable signals for the future.

The ATLAS and CMS Collaborations, while collecting data at 7, 8, and $13 \mathrm{TeV}$, performed searches for VLQs with different quantum numbers, probing single and pair production mechanisms, as well as decay modes into all three generations of SM quarks (for the most updated experimental results of ATLAS and CMS we refer to the respective web pages [3-5]). However, new extra quarks can be charged under new symmetries, such as $T$-parity in little Higgs [6-12] and Kaluza-Klein parity in extra dimension [13-17] models. Such VLQs have been searched for at both the Tevatron $[18,19]$ and the LHC [20,21], though no evidence for the existence of other quarks, beside those of the SM, has been obtained. Direct bounds on heavy chiral quarks can be interpreted as bound on VLQs, but it must be stressed that decay channels of VLQs are different from decay channels of heavy chiral quarks [22]. Thus, if the VLQs have a strong mass degeneracy, the visible decay products of the VLQ are too soft to be detected, and as a consequence, the bounds on the VLQ mass can be very weak, analogous to the case of strong degeneracy between squarks and neutralinos in supersymmetry. Intriguingly, as we shall detail below, even in our simple scenario, VLQ mass values down to $400 \mathrm{GeV}$ are still possible, so that they could strongly affect, e.g., the $g g \rightarrow h \rightarrow V \gamma$ (with $V=\gamma, Z$ ) rates.

Now, let us also assume that additional (pseudo)scalar objects possibly behind the LHC experimental data do originate from the same EWSB mechanism governing the generation of the $\approx 125 \mathrm{GeV}$ Higgs state. This is indeed a possibility not excluded by current theoretical and experimental constraints. Under these circumstances, it is then of phenomenological importance to consider the case of a second Higgs doublet participating in EWSB alongside the one responsible for the discovered Higgs state. This mass generation dynamics is well known in the form of $2 \mathrm{HDMs}$ [23]. We are therefore left with a new physics construct that would include a $2 \mathrm{HDM}$ supplemented by one or more VLQs as a potential scenario that could accommodate the LHC data on the $\approx 125 \mathrm{GeV}$ Higgs boson and additionally explain results above the SM yield.

In this paper, we wish to build on the results of [24-27], where a similar possibility was discussed (by some of us), in which the role of a $2 \mathrm{HDM}$ was played by a SM-like Higgs doublet supplemented by an additional Higgs singlet. We intend to review here a $2 \mathrm{HDM}$ plus single VLQ scenario, where the VLQ has the same electromagnetic (EM) charge of the top quark (with which it then mixes), as a candidate to study the implications of the heavy Higgs searches by the ATLAS and CMS Collaborations both with $8 \mathrm{TeV}$ and the latest $13 \mathrm{TeV}$ data. Furthermore, we will relate such data samples to those involving $\gamma \gamma$ and $Z \gamma$ final states. In addition, we will show an enhancement of the $p p \rightarrow t \bar{t} h$ cross section at the LHC induced by small mixing of the top quark with the additional state $T$. Finally, we will discuss the possibility of VLQs produced as real objects in the detector decaying into Higgs boson states, both neutral and charged.

Our paper is formatted as follows. In the next section, we describe in some detail the model concerned. In the three subsequent sections, we present our results, followed by our conclusions.

\section{A 2HDM EXTENDED BY AN UP-TYPE VECTORLIKE QUARK}

A simple extension of the SM is the well-known 2HDM that expands the Higgs sector of the SM by an additional Higgs doublet. The spectrum of the model contains additional Higgses and possesses an alignment limit [28], in which one of the Higgses completely mimics the SM one.

To describe our model, we start with the well known $C P$-conserving $2 \mathrm{HDM}$ scalar potential for $\left(\Phi_{1}, \Phi_{2}\right)$ with a discrete symmetry $\Phi_{1} \rightarrow-\Phi_{1}$ that is only violated softly by dimension two terms $[23,29]$ :

$$
\begin{aligned}
V( & \left.\Phi_{1}, \Phi_{2}\right) \\
= & m_{11}^{2} \Phi_{1}^{\dagger} \Phi_{1}+m_{22}^{2} \Phi_{2}^{\dagger} \Phi_{2}-m_{12}^{2}\left(\Phi_{1}^{\dagger} \Phi_{2}+\Phi_{2}^{\dagger} \Phi_{1}\right) \\
& +\frac{\lambda_{1}}{2}\left(\Phi_{1}^{\dagger} \Phi_{1}\right)^{2}+\frac{\lambda_{2}}{2}\left(\Phi_{2}^{\dagger} \Phi_{2}\right)^{2}+\lambda_{3}\left(\Phi_{1}^{\dagger} \Phi_{1}\right)\left(\Phi_{2}^{\dagger} \Phi_{2}\right) \\
& +\lambda_{4}\left(\Phi_{1}^{\dagger} \Phi_{2}\right)\left(\Phi_{2}^{\dagger} \Phi_{1}\right)+\frac{\lambda_{5}}{2}\left[\left(\Phi_{1}^{\dagger} \Phi_{2}\right)^{2}+\left(\Phi_{2}^{\dagger} \Phi_{1}\right)^{2}\right],
\end{aligned}
$$

where all parameters are real. The two complex scalar doublets $\Phi_{1,2}$ may be rotated into a basis, $H_{1,2}$, where only one obtains a vacuum expectation value (VEV),

$$
H_{1}=\left(\begin{array}{c}
G^{+} \\
v+\varphi_{1}^{0}+i G^{0} \\
\sqrt{2}
\end{array}\right), \quad H_{2}=\left(\begin{array}{c}
H^{+} \\
\frac{\varphi_{2}^{0}+i A}{\sqrt{2}}
\end{array}\right),
$$

where $G^{0}$ and $G^{ \pm}$are the would-be Goldstone bosons and $H^{ \pm}$are a pair of charged Higgses. Herein, $A$ is a $C P$-odd pseudoscalar which does not mix with the other neutral states in the $C P$-conserving case. The physical $C P$-even scalars $h$ and $H$ are mixtures of $\varphi_{1,2}^{0}$, and the scalar mixing is parametrized as ${ }^{1}$

$$
\left(\begin{array}{c}
h \\
H
\end{array}\right)=\left(\begin{array}{cc}
s_{\beta-\alpha} & c_{\beta-\alpha} \\
c_{\beta-\alpha} & -s_{\beta-\alpha}
\end{array}\right)\left(\begin{array}{c}
\varphi_{1}^{0} \\
\varphi_{2}^{0}
\end{array}\right),
$$

\footnotetext{
${ }^{1}$ Hereafter, $s_{X} \equiv \sin X$ and $c_{X}=\cos X$.
} 
where $\tan \beta=v_{2} / v_{1}$ is the angle used to rotate $\Phi_{1,2}$ into $H_{1,2}$ and $\alpha$ is the additional mixing needed to diagonalize the $C P$-even mass matrix. As mentioned in the Introduction, in order to alter the gluon-gluon-Higgs, photon-photon-Higgs, and/or Z-photon-Higgs couplings, one can advocate the inclusion of new heavy fermions such as a VLQ partner of the top quark with the same EM charge. In fact, there are many SM extensions that require vectorlike fermions in their spectrum (for an overview see $[25,30])$. Such a new VLQ will mix with the top quark through the Yukawa interactions and can contribute, therefore, to some SM observables. To derive these new interactions, we first study the Yukawa sector within a 2HDM extended by a VLQ pair $\left(T_{L}, T_{R}\right)$ in the $\mathbf{1}_{2 / 3}$ representation of the SM EW group. In the 2HDM-II, our concern here, one doublet couples to up quarks and the other one couples to down quarks and charged leptons. The most general renormalizable model for the quark Yukawa interactions and mass terms can be described, limited to third generation quarks and new VLQs, by the following Lagrangian:

$$
\begin{aligned}
& -\mathcal{L}_{Y}^{I I} \supset y_{T} \overline{Q_{L}^{0}} \tilde{H}_{2} T_{R}^{0}+\xi_{T} \overline{Q_{L}^{0}} \tilde{H}_{1} T_{R}^{0}+M_{T} \overline{T_{L}^{0}} T_{R}^{0} \\
& \quad \supset y_{T}\left(\overline{t_{L}^{0}}, \overline{b_{L}^{0}}\right)\left(\begin{array}{c}
\frac{\varphi_{2}^{0}-i A}{\sqrt{2}} \\
-H^{-}
\end{array}\right) T_{R}^{0}+\xi_{T}\left(\overline{t_{L}^{0}}, \overline{b_{L}^{0}}\right)\left(\begin{array}{c}
\frac{v+\varphi_{1}^{0}-i G^{0}}{\sqrt{2}} \\
-G^{-}
\end{array}\right) T_{R}^{0} \\
& \quad+M_{T} \overline{T_{L}^{0}} T_{R}^{0}+\text { H.c }
\end{aligned}
$$

where $\tilde{H}_{i} \equiv i \tau_{2} H_{i}^{*}(i=1,2), Q_{L}^{0}$ are the SM quark doublets, and the $u_{R}^{i}$,s are the SM up-type quark singlets. Note that additional kinetic mixing terms of the form $\overline{T_{L}} t_{R}^{i}$ can always be rotated away and reabsorbed into the definition of $y_{t, T}$. Furthermore, one can, without loss of generality, choose a weak interaction basis where $y_{t}$ is diagonal and real. In the weak eigenstate basis $\left(t_{L}^{0}, T_{L}^{0}\right)$, the top quark and VLQ mass matrix is

$$
\mathcal{M}=\left(\begin{array}{cc}
\frac{y_{t} v}{\sqrt{2}} & \frac{\xi_{T} v}{\sqrt{2}} \\
0 & M_{T}
\end{array}\right)
$$

where $y_{t}$ and $\xi_{T}$ are the Yukawa couplings for the top quark and VLQ, respectively, $v=246 \mathrm{GeV}$ is the VEV of the SM Higgs doublet, while $M_{T}$ is a bare mass term of the VLQ, which, as intimated, is unrelated to the Higgs mechanism of EWSB. It is clear from the above mass matrix that the physical mass of the heavy top, $m_{T}$, is different from $M_{T}$ due to the $t-T$ mixing. Furthermore, such a mass matrix can be diagonalized by a bi-unitary transformation such that

$$
U_{L} \mathcal{M} U_{R}^{\dagger}=\mathcal{M}_{d}, \quad U_{R, L} \mathcal{M}^{\dagger} \mathcal{M} U_{R, L}^{\dagger}=\mathcal{M}_{d}^{2},
$$

with $\mathcal{M}$ the matrix given in Eq. (5) and $\mathcal{M}_{d}$ the diagonalized one. The unitary matrices $U_{L}$ and $U_{R}$ are defined by

$$
\left(\begin{array}{c}
t_{L, R} \\
T_{L, R}
\end{array}\right)=\left(\begin{array}{cc}
\cos \left(\theta_{L, R}\right) & -\sin \left(\theta_{L, R}\right) \\
\sin \left(\theta_{L, R}\right) & \cos \left(\theta_{L, R}\right)
\end{array}\right)\left(\begin{array}{c}
t_{L, R}^{0} \\
T_{L, R}^{0}
\end{array}\right) .
$$

In fact, the mixing angles $\theta_{L}$ and $\theta_{R}$ are not independent parameters. From the bi-unitary transformations applied to Eq. (6), we can derive the following relations:

$$
\begin{aligned}
\tan \left(2 \theta_{L}\right) & =\frac{4 m_{t} M_{T}}{2 M_{T}^{2}-2 m_{t}^{2}-\xi_{T}^{2} v^{2}}, \\
\tan \left(2 \theta_{R}\right) & =\frac{2 \sqrt{2} \xi_{T} m_{t} v}{2 M_{T}^{2}+2 m_{t}^{2}-\xi_{T}^{2} v^{2}} .
\end{aligned}
$$

The above equations in turn give the following relationships between $\theta_{L}$ and $\theta_{R}$ (see [25]):

$$
\tan \theta_{L}=\frac{m_{t}}{m_{T}} \tan \theta_{R}, \quad \frac{\xi_{T}}{y_{t}}=s_{L} c_{L} \frac{m_{T}^{2}-m_{t}^{2}}{m_{t} m_{T}} .
$$

After rotating the weak eigenstates $\left(t_{L}^{0}, T_{L}^{0}\right)$ into the mass eigenstates, the Yukawa Lagrangian takes the following form:

$$
\begin{aligned}
-\mathcal{L}_{Y}^{I I} \supset & \left(\overline{t_{L}}, \bar{T}_{L}\right) U_{L}\left[\varphi_{1}^{0}\left(\begin{array}{cc}
\frac{y_{t}}{\sqrt{2}} & \frac{\xi_{T}}{\sqrt{2}} \\
0 & 0
\end{array}\right)\right. \\
& \left.+\varphi_{2}^{0}\left(\begin{array}{cc}
\frac{y_{t}}{\sqrt{2}} \xi_{u} & \frac{y_{T}}{\sqrt{2}} \\
0 & 0
\end{array}\right)\right] U_{R}^{\dagger}\left(\begin{array}{c}
t_{R} \\
T_{R}
\end{array}\right) \\
& -i\left(\overline{t_{L}}, \bar{T}_{L}\right) A\left[U_{L}\left(\begin{array}{cc}
\frac{y_{t}}{\sqrt{2}} \xi_{u} & \frac{y_{T}}{\sqrt{2}} \\
0 & 0
\end{array}\right) U_{R}^{\dagger}\right]\left(\begin{array}{c}
t_{R} \\
T_{R}
\end{array}\right)+\text { H.c. }
\end{aligned}
$$

The neutral Higgs couplings to top $(t)$ and heavy top $(T)$ quark pairs normalized to the $h_{\mathrm{SM}} t \bar{t}$ one are given in Appendix A.

In our $2 \mathrm{HDM}+\mathrm{VLQ}$ scenario, neutral and charged current interactions receive contributions from the new VLQ,

$$
\begin{aligned}
\mathcal{L}= & -\frac{g}{\cos \theta_{W}} Z_{\mu} \bar{f} \gamma^{\mu}\left(g_{f f^{\prime}}^{L} P_{L}+g_{f f^{\prime}}^{R} P_{R}\right) f^{\prime} \\
& +\frac{g}{\sqrt{2}}\left(V_{t b} \bar{t}+V_{T b} \bar{T}\right) \gamma^{\mu} P_{L} b W_{\mu}^{+}+\text {H.c. },
\end{aligned}
$$

with $f, f^{\prime}=t, T$. The new couplings are modified as follows:

$$
\begin{gathered}
g_{t t}^{L}=T_{3}^{t}-Q_{t} \sin ^{2} \theta_{W}-\frac{s_{L}^{2}}{2}, \\
g_{T T}^{L}=T_{3}^{T}-Q_{T} \sin ^{2} \theta_{W}-\frac{c_{L}^{2}}{2},
\end{gathered}
$$

$g_{t T}^{L}=\frac{s_{L} c_{L}}{2}, \quad g_{t t}^{R}=g_{T T}^{R}=-Q_{t} \sin ^{2} \theta_{W}, \quad g_{t T}^{R}=0$, 
TABLE I. The Higgs signal strengths in various production and decay channels measured by ATLAS and CMS presented in combination at both LHC Run 1 (combined $\sqrt{s}=7$ and $8 \mathrm{TeV}$ ) and Run $2(\sqrt{s}=13 \mathrm{TeV})$.

\begin{tabular}{lccc}
\hline \hline & \multicolumn{2}{c}{ LHC data } \\
\cline { 2 - 4 } & & & Run 2 \\
\cline { 2 - 4 } Higgs signal strength & Run 1 [32] & ATLAS [34-36] & CMS [37-39] \\
\hline$\hat{\mu}_{1}^{\gamma \gamma}$ & $1.10_{-0.21}^{+0.23}$ & $0.67_{-0.21}^{+0.25}$ & $0.77_{-0.23}^{+0.25}$ \\
$\hat{\mu}_{2}^{\gamma \gamma}$ & $0.8_{-0.71}^{+.71}$ & $2.25_{-0.75}^{+0.75}$ & $1.59_{-0.45}^{+0.73}$ \\
$\hat{\mu}_{1}^{Z Z}$ & $1.27_{-0.24}^{+0.28}$ & $1.42_{-0.31}^{+0.35}$ & $1.20_{-0.21}^{+0.22}$ \\
$\hat{\mu}_{2}^{Z Z}$ & $1.66_{-0.44}^{+0.51}$ & $3.8_{-2.2}^{+2.8}$ & $0.67_{-0.67}^{+1.61}$ \\
$\hat{\mu}_{1}^{W W}$ & $1.06_{-0.18}^{+0.21}$ & $\ldots$ & $\ldots$ \\
$\hat{\mu}_{2}^{W W}$ & $1.27_{-0.45}^{+0.53}$ & $\ldots$ & $\ldots$ \\
$\hat{\mu}_{1}^{b \bar{b}}$ & $0.64_{-0.28}^{+0.37}$ & $3.9_{-2.9}^{+2.8}$ & $3.7_{-2.5}^{+2.4}$ \\
$\hat{\mu}_{2}^{b \bar{b}}$ & $0.51_{-0.37}^{+0.40}$ & & $\ldots$ \\
$\hat{\mu}_{1}^{\tau \tau}$ & $1.05_{-0.27}^{+0.33}$ & $\ldots$ & $\ldots$ \\
$\hat{\mu}_{2}^{\tau \tau}$ & $1.24_{-0.54}^{+0.58}$ & $\ldots$ & $\ldots$ \\
\hline \hline
\end{tabular}

$$
V_{T b}=s_{L}, \quad \text { and } \quad V_{t b}=c_{L}
$$

Finally, the interaction with the charged scalar boson and the new quark $T$ can be written as

$$
\mathcal{L}=-\frac{g V_{T b}}{\sqrt{2} M_{W}} \bar{T}\left(\frac{m_{T}}{\tan \beta} P_{L}+m_{b} \tan \beta P_{R}\right) b H^{+}+\text {H.c. }
$$

\section{RESULTS AND DISCUSSIONS}

In our numerical calculation, we consider the scenario with a light Higgs boson $h$ as the SM-like state, with $m_{h}=125 \mathrm{GeV}$. We take into account theoretical constraints from vacuum stability, unitarity, and perturbativity. We then enforce bounds from precision EW data (such as the oblique parameters $S, T$, and $U$ ) and adopt constraints on the charged Higgs boson mass from the 2HDM-II using $b \rightarrow s \gamma$ rates, which set a limit $m_{H^{ \pm}}>580 \mathrm{GeV}$ [31].

In addition, we perform a global $\chi^{2}$ analysis for the signal strengths of the observed Higgs boson $h$ from the combined production modes $i=1(\mathrm{ggh}+\mathrm{tth})$ as well as $i=2$ $(\mathrm{VBF}+\mathrm{Vh})$ and decay modes into $f=\gamma \gamma, Z Z, W^{+} W^{-}$, $\tau^{-} \tau^{+}$, and $b \bar{b}$ [32],

$$
\chi^{2}=\sum_{i=1,2} \frac{\left(\hat{\mu}_{i}^{f}-\mu_{i}^{f}\right)^{2}}{\Delta \mu_{i}^{f 2}},
$$

where the signal strength variable $\mu_{i}^{f}$ is defined as

$$
\mu_{i}^{f}=\frac{\sigma(i \rightarrow h) \mathrm{BR}(h \rightarrow f)}{\sigma_{\mathrm{SM}}(i \rightarrow h) \mathrm{BR}_{\mathrm{SM}}(h \rightarrow f)},
$$

in terms of a production cross section, $\sigma$, and a decay branching ratio (BR). The parameters with the subscript "SM" represent the corresponding values for the SM. The experimentally obtained best-fit signal strength values which we have implemented in our analysis are given in Table I. Furthermore, in Eq. (17), $\Delta \mu_{i}^{f 2}$ represents the error associated with the experimental measurement. We use HigGsBounds-4.3.1 [33] to constrain the nonobservation of neutral and/or charged Higgs bosons at the LHC at $95 \%$ C.L.

\section{A. Constraints on $m_{T}$}

As intimated in the Introduction, ATLAS and CMS have performed direct searches for VLQs at 7,8 TeV, having potential sensitivities up to $800 \mathrm{GeV}$ or so [25,40-42]. We have already explained that several VLQ scenarios may be conceived in order to enable $m_{\mathrm{VLQ}}$ values down to $350 \mathrm{GeV}$ or so yet still be compatible with data. Clearly, the decay patterns of new VLQs depend on the representation of these fermionic states. In our rather simple scenario, i.e., in the case of a singlet VLQ, if we neglect the first and second generation mixing, the heavy top $T$ will decay into the following final states: $W^{+} b, Z t$, and $h t$, where, as explained, $h$ now plays the role of the SM-like Higgs state. Under these assumptions, the ATLAS search in Ref. [43] is the most constraining one and excludes a heavy $T$ quark with mass lower than $\approx 640 \mathrm{GeV}$ at the $95 \%$ confidence level (C.L.). This lower limit can, however, be weakened down to $\approx 350 \mathrm{GeV}$ if $T$ couples to first and second generation quarks as well [44]. This is certainly a possible model construction; however, in our case, we do not pursue this in any detail, as such additional interactions would not enter the Higgs boson observables which we intend to study. We are nonetheless entitled to scan on $m_{T}$ starting from such low $m_{\mathrm{VLQ}}$ values. In our $2 \mathrm{HDM}+\mathrm{VLQ}$ 
construct, if we assume $m_{A}, m_{H}, m_{H^{ \pm}}<m_{T}-m_{t}$, then also the $T \rightarrow t H, T \rightarrow t A$, and $T \rightarrow b H^{ \pm}$decays open up, alongside $T \rightarrow t h$. Finally, one should recall that $T$ production at the LHC is substantial, in both the QCD induced pair production channel (dominant at low $m_{T}$ ) and the EW mediated single production channel (dominant at high $m_{T}$ ).

\section{B. Constraints on the $\boldsymbol{t}-\boldsymbol{T}$ mixing}

In this section, we will show that $t-T$ mixing can be constrained both from EW precision observables (EWPOs) and from recent LHC data on the $\approx 125 \mathrm{GeV}$ Higgs boson. In general, when the new physics scale is much larger than the EW scale, virtual effects of the new particles in loops are expected to contribute to the EWPOs that have been precisely measured at LEP1, LEP2, SLC, and Tevatron. These EWPOs are known as the oblique parameters $S, T$, and $U$ [45] and can be used to put constraints on new physics. In our case, the mixing between $t-T$ will generate couplings between the SM gauge bosons and the new VLQ, $T$, which will induce contributions to $S$ and $T$ [46].

We have computed the extra contributions of the VLQ to $\Delta T=T-T_{\mathrm{SM}}$ and $\Delta S=S-S_{\mathrm{SM}}$ by implementing the model into the FeynArTs [47], FormCALC [48,49], and LoopTools [50,51] packages, which are used to calculate the required gauge boson self-energies. In fact, in our case, the extra contribution to $\Delta\{T, S\}$ can be cast

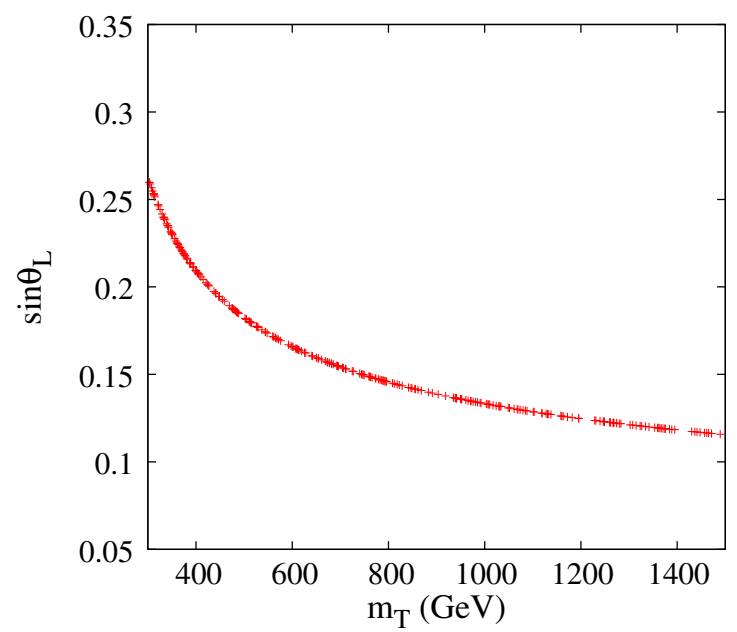

FIG. 1. Upper limit at $95 \%$ C.L. on the (left-handed) mixing angle as a function of the $T$ quark mass in the 2HDM-II with an uplike VLQ.

into pure 2HDM and VLQ parts, such that $\Delta\{T, S\}=$ $\Delta\{T, S\}_{2 \mathrm{HDM}}+\Delta\{T, S\}_{\mathrm{VLQ}}$. In the present work, we focus on the decoupling limit where $m_{A}=m_{H}=m_{H^{ \pm}} \gg m_{Z}$ and $\sin (\beta-\alpha)=1$, or slight departures from it, which leads to $\Delta T_{2 \mathrm{HDM}}=0$ and $\Delta S_{2 \mathrm{HDM}}=0$. We are then left with only the extra contribution of the VLQ. A straightforward calculation yields

$$
\begin{aligned}
\Delta T_{\mathrm{VLQ}} & =\frac{3 m_{t}^{2} s_{L}^{2}}{16 m_{W}^{2} \pi(-1+r) s_{W}^{2}}\left((-1+r)\left(-2+(1+r) s_{L}^{2}\right)-2 r c_{L}^{2} \log r\right) \quad \text { with } r=\frac{m_{T}^{2}}{m_{t}^{2}}, \\
\Delta S_{\mathrm{VLQ}}= & \frac{1}{12 m_{Z}^{2} \pi}\left\{-m_{t}^{2} s_{L}^{2}+m_{T}^{2}\left(s_{L}^{2}+32 s_{W}^{2} c_{W}^{2}\right)+s_{L}^{2}\left(m_{Z}^{2}\left(10-9 s_{L}^{2}\right)-6 m_{t}^{2} c_{L}^{2}+6 m_{T}^{2} c_{L}^{2}\right) A_{0}\left[m_{t}^{2}\right]\right. \\
& +\left(s_{L}^{2}\left(6 m_{t}^{2} c_{L}^{2}-6 m_{T}^{2} c_{L}^{2}+m_{Z}^{2}\left(-10+9 s_{L}^{2}\right)\right)-32 m_{Z}^{2} s_{W}^{2} c_{W}^{2}\right) A_{0}\left(m_{T}^{2}\right) \\
& +3 m_{t}^{2} s_{L}^{2}\left(10-3 s_{L}^{2}\right) B_{0}\left(0, m_{t}^{2}, m_{t}^{2}\right)-18 m_{t}^{2} s_{L}^{2} c_{L}^{2} B_{0}\left(0, m_{t}^{2}, m_{T}^{2}\right) \\
& -m_{T}^{2}\left(12 s_{L}^{2}+9 s_{L}^{4}-32 s_{W}^{2} c_{W}^{2}\right) B_{0}\left(0, m_{T}^{2}, m_{T}^{2}\right) \\
& +2 s_{L}^{2}\left(m_{Z}^{2}\left(2-3 s_{L}^{2}\right)+m_{t}^{2}\left(-14+3 s_{L}^{2}\right)\right) B_{0}\left[m_{Z}^{2}, m_{t}^{2}, m_{t}^{2}\right] \\
& +6\left(\left(m_{t}^{2}-m_{T}^{2}\right)^{2}+\left(m_{t}^{2}+m_{T}^{2}\right) m_{Z}^{2}-2 m_{Z}^{4}\right) s_{L}^{2} c_{L}^{2} B_{0}\left[m_{Z}^{2}, m_{t}^{2}, m_{T}^{2}\right] \\
& \left.+2 s_{L}^{2}\left(m_{Z}^{2}\left(4-3 s_{L}^{2}\right)+m_{T}^{2}\left(8+3 s_{L}^{2}\right)\right) B_{0}\left[m_{Z}^{2}, m_{T}^{2}, m_{T}^{2}\right]\right\},
\end{aligned}
$$

where the $A_{0}$ and $B_{0}$ functions are the standard PassarinoVeltman ones used in the convention of LoOPToOLS [51]. Note that our results agree numerically with Ref. [52]. Taking the above analytical expressions into account, our model will remain viable as long as $\Delta T_{\mathrm{VLQ}}$ and $\Delta S_{\mathrm{VLQ}}$ are compatible with the latest extracted values [53] which are given by

$$
\Delta T=0.1 \pm 0.07, \quad \Delta S=0.06 \pm 0.09,
$$

where a correlation coefficient $\rho=+0.91$ and $\Delta U=0$ have been used. We thus perform a random scan on the $s_{L}$ and $m_{T}$ parameters imposing compatibility with $\Delta T$ and $\Delta S$ at $95 \%$ C.L., which yields a constraint on $s_{L}$ as a function of the VLQ mass, $m_{T}$, as shown in Fig. 1. One can see that the constraint on the mixing is, e.g., $\left|s_{L}\right| \leq 0.25$ for $m_{T}=350 \mathrm{GeV}$ and $\left|s_{L}\right| \leq 0.12$ for $m_{T}=1 \mathrm{TeV}$. For a heavy VLQ, the constraint on the mixing is more severe and is mainly coming from $\Delta T$ which contains a large logarithm of $m_{T}^{2} / m_{t}^{2}$. 


\section{Constraints from $B \rightarrow X_{s} \gamma$}

In addition to the EWPO constraints studied above, the penguin induced $b \rightarrow s \gamma$ decay is also sensitive to new physics. The current experimental value is $\operatorname{BR}(\bar{B} \rightarrow$ $\left.X_{s} \gamma\right)^{\exp }=(3.32 \pm 0.15) \times 10^{-4}$, for $E_{\gamma}>1.6 \mathrm{GeV}$ [54], and the SM prediction with next-to-next-to-leading order (NNLO) QCD corrections is $\operatorname{BR}\left(\bar{B} \rightarrow X_{s} \gamma\right)^{\mathrm{SM}}=(3.36 \pm$ $0.23) \times 10^{-4}[55,56]$. Since the SM result is close to the experimental data, $\bar{B} \rightarrow X_{s} \gamma$ will give a strict bound on new physics effects. The effective Hamiltonian arising from the $W^{ \pm}$and $H^{ \pm}$bosons for $b \rightarrow s \gamma$ at the $\mu_{b}=4.8 \mathrm{GeV}$ scale can be written as

$\mathcal{H}_{b \rightarrow s \gamma}=-\frac{4 G_{F}}{\sqrt{2}} \sum_{i=t, T} V_{i s}^{*} V_{i b}\left(C_{7 \gamma}^{i}\left(\mu_{b}\right) O_{7 \gamma}+C_{8 \gamma}^{i}\left(\mu_{b}\right) Q_{8 G}\right)$,

where the EM and gluonic dipole operators are given as

$$
\begin{aligned}
O_{7 \gamma} & =\frac{e}{16 \pi^{2}} m_{b} \bar{s} \sigma^{\mu \nu} P_{R} b F_{\mu \nu}, \\
O_{8 G} & =\frac{g_{s}}{16 \pi^{2}} m_{b} \bar{s}_{\alpha} \sigma^{\mu \nu} T_{\alpha \beta}^{a} P_{R} b_{\beta} G_{\mu \nu}^{a} .
\end{aligned}
$$

Here, $C_{7 \gamma}\left(\mu_{b}\right)$ and $C_{8 G}\left(\mu_{b}\right)$ are the Wilson coefficients at the $\mu_{b}$ scale, and their relations to the initial conditions at the high energy scale $\mu_{H}$ (needed to describe the evolution from such a high scale down to the lower energy $\mu_{b}$ via the matching scale $\mu_{0}$ [57]) are through renormalization group equations (RGEs). The NLO [57-59] and NNLO [60] QCD corrections to $C_{7 \gamma}\left(\mu_{b}\right)$ and $C_{8 G}\left(\mu_{b}\right)$ in the 2HDM-II have been calculated. Based on the $C_{7 \gamma}^{\mathrm{SM}}\left(\mu_{b}\right)$ value extracted in [61], we get $C_{7 \gamma}^{\mathrm{SM}}\left(\mu_{b}\right) \approx-0.310$ when $\operatorname{BR}\left(\bar{B} \rightarrow X_{s} \gamma\right)^{\mathrm{SM}}=$ $3.36 \times 10^{-4}$ is applied. In order to study the influence of the

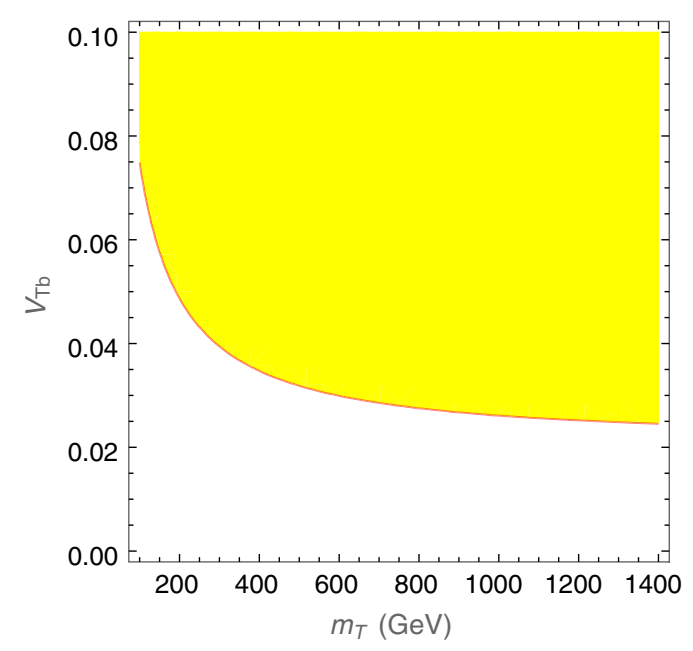

$b \rightarrow s \gamma$ process on the $2 \mathrm{HDM}+$ VLQ model, we follow the approach in [56] and split the $\operatorname{BR}\left(\bar{B} \rightarrow X_{s} \gamma\right)$ as follows:

$$
\begin{aligned}
\operatorname{BR}\left(\bar{B} \rightarrow X_{s} \gamma\right) \times 10^{4} \approx & (3.36 \pm 0.23)-8.22 \operatorname{Re}\left(C_{7 \gamma}^{i}\right) \\
& -1.99 \operatorname{Re}\left(C_{8 G}^{i}\right),
\end{aligned}
$$

where $C_{7 \gamma, 8 G}^{i}$ are the Wilson coefficients at the $\mu_{H}=m_{H^{ \pm}}$ scale (the matching scale is $\mu_{0} \sim m_{t}$ at which the heavy particles are decoupled [56]), wherein the quadratic $C_{7 \gamma, 8 G}^{i}$ terms are ignored due to the requirement of $C_{7 \gamma, 8 G}^{i}<1$. Using the current experimental value, the bound on $C_{7 \gamma, 8 G}^{i}$ is

$$
8.22 \operatorname{Re}\left(C_{7 \gamma}^{i}\right)+1.99 \operatorname{Re}\left(C_{8 G}^{i}\right) \approx 0.04 \pm 0.28 .
$$

According to the charged Higgs interactions, the $H^{ \pm}$ contributions coming together with $t$ and $T$ to $C_{7 \gamma, 8 G}^{i}$ are expressed as [57]

$$
\begin{gathered}
C_{7 \gamma}^{i}=f_{1 \gamma}\left(x_{i}\right) / \tan ^{2} \beta+f_{2 \gamma}\left(x_{i}\right), \\
C_{8 G}^{i}=f_{1 G}\left(x_{i}\right) / \tan ^{2} \beta+f_{2 G}\left(x_{i}\right),
\end{gathered}
$$

with $x_{i}=m_{i}^{2} / m_{H^{ \pm}}^{2}$ and $i=t, T$. The form factors are given in Appendix B.

Figure 2 shows the limits on $V_{T b}$ (left) and $m_{H^{ \pm}}$(right) in the $2 \mathrm{HDM}+\mathrm{VLQ}$. We learn that $\mathrm{BR}(b \rightarrow s \gamma)$ excludes $V_{T b} \geq 0.03$ regardless of the value of $m_{T}$ used. It further appears from the right panel of Fig. 2 that large $\tan \beta$ is not excluded by current data and a lower bound $m_{H^{ \pm}} \geq$ $600 \mathrm{GeV}$ is obtained similar to the case of the standard 2HDM-II. (Recall that a lower bound $m_{H^{ \pm}} \geq 580 \mathrm{GeV}$ was obtained in [31] for this case.)

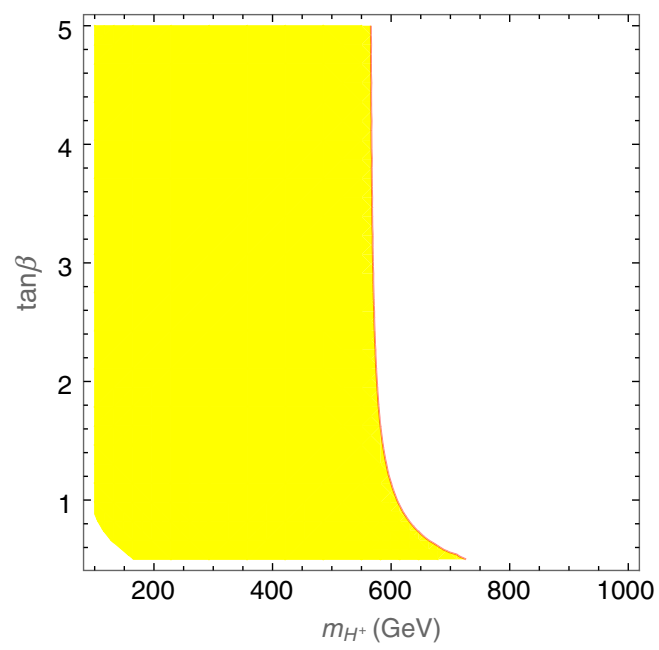

FIG. 2. Excluded regions (yellow color) at 95\% C.L. from $\mathrm{BR}\left(\bar{B} \rightarrow X_{s} \gamma\right)$ in the 2HDM + VLQ. Left panel: Limit on $V_{T b}$ as a function of $m_{T}$ with $m_{H^{ \pm}}=600 \mathrm{GeV}$. Right panel: Limit on the charged Higgs mass with $m_{T}=400 \mathrm{GeV}$ and $V_{T b}=0.025$. 

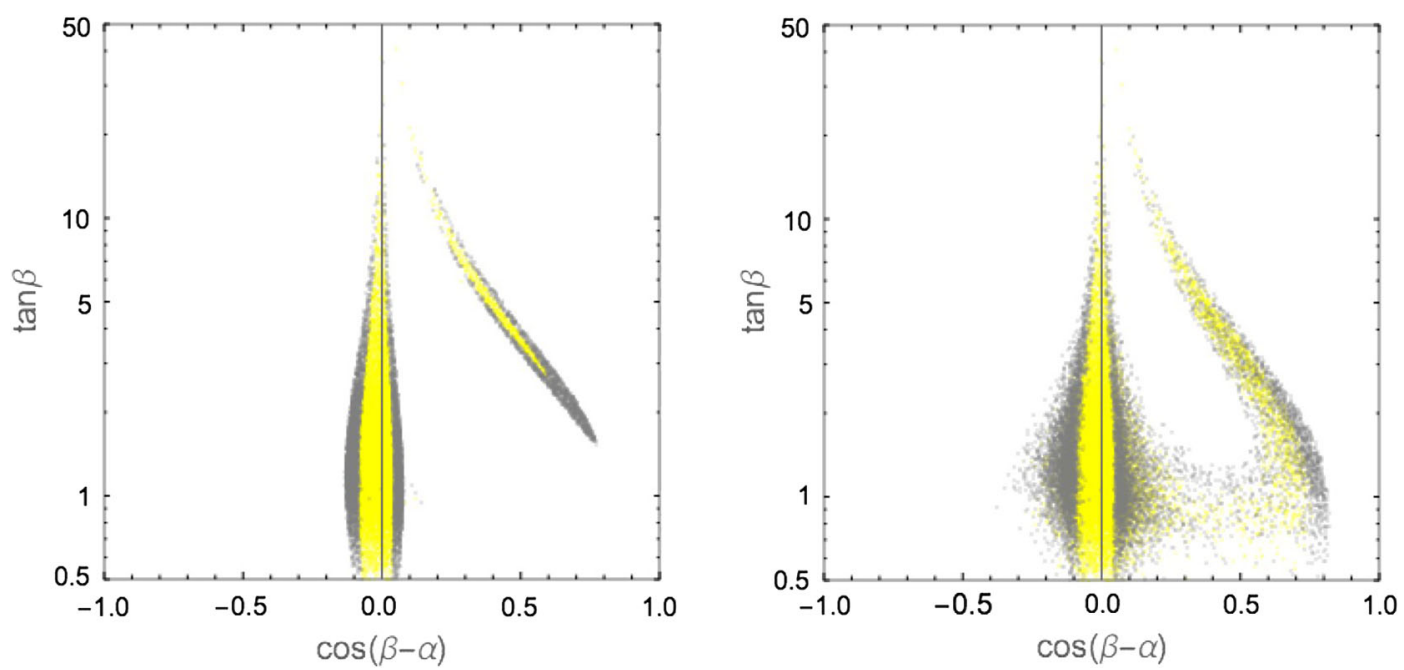

FIG. 3. Constraints from the LHC Higgs data on the parameter space of the ordinary 2HDM (left) and 2HDM + VLQ (right). We show the $95 \%$ C.L. region in the $[\cos (\beta-\alpha), \tan \beta]$ plane. The gray (yellow) regions were obtained using Higgs Run 1 (2) data. We have varied $\left|s_{L}\right|<0.20$ and $400 \mathrm{GeV}<m_{T}<1000 \mathrm{GeV}$ and set $y_{T}=4 \pi$.

\section{Constraints from LHC data}

The couplings of the SM-like Higgs are sensitive to the parameters $\cos (\beta-\alpha)$ and $\tan \beta$. Therefore, the LHC data on the $125 \mathrm{GeV}$ Higgs boson can give strong constraints on these. In Fig. 3, we show the constraints on the ordinary 2HDM (left) and 2HDM + VLQ (right) using Higgs data from Run 1 (gray) and Run 2 (yellow) at 95\% C.L. The bounds on $\cos (\beta-\alpha)$ are much more stringent for the ordinary 2HDM-II, where the SM-like coupling region of the $125 \mathrm{GeV}$ Higgs forces $|\cos (\beta-\alpha)|<0.14$, and increasingly more stringent for larger $\tan \beta$. However, in the so-called "wrong sign" Yukawa coupling region of the
$125 \mathrm{GeV}$ Higgs state, we find $|\cos (\beta-\alpha)|<0.45$. By varying $\left|s_{L}\right|<0.20$ and $400 \mathrm{GeV}<m_{T}<1000 \mathrm{GeV}$ and setting $y_{T}=4 \pi$, the situation in the $2 \mathrm{HDM}+\mathrm{VLQ}$ (right panel) is quite different for low $\tan \beta$, where $|\cos (\beta-\alpha)|<0.4$.

Another constraint, this time on the mixing, $s_{L}$, comes from the contribution of the VLQ to the diphoton event rate of the $\approx 125 \mathrm{GeV}$ SM-like Higgs boson. The modified top quark coupling to this Higgs boson and the presence of an additional heavy quark can impact loop induced Higgs decays, namely, $h \rightarrow g g, h \rightarrow \gamma \gamma$, and $h \rightarrow Z \gamma$. The relevant partial decay widths are given $b^{2}$

$$
\begin{aligned}
\Gamma(\phi \rightarrow \gamma \gamma)= & \frac{G_{F} \alpha^{2} m_{\phi}^{3}}{128 \sqrt{2} \pi^{3}}\left|\frac{4}{3} \sum_{f=t, T} \kappa_{f f}^{\phi} A_{1 / 2}^{\phi}\left(\tau_{f}\right)+\kappa_{W W}^{\phi} A_{1}^{\phi}\left(\tau_{W}\right)+g_{H^{ \pm} H^{\mp}}^{\phi} \frac{m_{W}^{2}}{2 c_{W}^{2} m_{H^{ \pm}}^{2}} A_{0}^{\phi}\left(\tau_{H^{ \pm}}\right)\right|^{2}, \\
\Gamma(\phi \rightarrow Z \gamma)= & \frac{G_{F}^{2} m_{W}^{2} \alpha m_{\phi}^{3}}{64 \pi^{4}}\left(1-\frac{m_{Z}^{2}}{m_{\phi}^{2}}\right)^{3} \mid \sum_{f=t, T} \kappa_{f f}^{\phi} \frac{2 n_{f} Q_{f}\left(I_{f}^{3}-2 s_{W}^{2} Q_{f}\right)}{c_{W}} A_{1 / 2}^{\phi}\left(\tau_{f}, \lambda_{f}\right) \\
& +\sum_{f, f^{\prime}=t, T} \frac{m_{f\left(f^{\prime}\right)}}{m_{W}} \sum_{m=L, R} \kappa_{f f^{\prime}}^{\phi} g_{f f^{\prime}}^{m} A_{1 / 2}^{\phi}\left(\tau_{f}, \lambda_{f^{\prime}}\right)+\kappa_{W W}^{\phi} A_{1}^{\phi}\left(\tau_{W}\right)+\left.g_{H^{ \pm} H^{\mp}}^{\phi} \frac{m_{W}^{2}}{m_{H^{ \pm}}^{2}} \frac{c_{2 W}}{c_{W}} A_{0}^{\phi}\left(\tau_{H^{ \pm}}\right)\right|^{2}, \\
\Gamma(A \rightarrow \gamma \gamma)= & \frac{G_{F} \alpha^{2} m_{A}^{3}}{128 \sqrt{2} \pi^{3}}\left|\frac{4}{3} \sum_{f=t, T} \kappa_{f f}^{A} A_{1 / 2}^{A}\left(\tau_{f}\right)\right|^{2}, \\
\Gamma(A \rightarrow Z \gamma)= & \frac{G_{F}^{2} m_{W}^{2} \alpha m_{A}^{3}}{16 \pi^{4}}\left(1-\frac{m_{Z}^{2}}{m_{A}^{2}}\right)^{3} \\
& \left.\times \mid \sum_{f=t, T} \kappa_{f f}^{A} \frac{2 n_{f} Q_{f}\left(I_{f}^{3}-2 s_{W}^{2} Q_{f}\right)}{c_{W}} A_{1 / 2}^{A}\left(\tau_{f}, \lambda_{f}\right)+\sum_{f, f^{\prime}=t, T} \frac{m_{f\left(f^{\prime}\right)}}{m_{W}} \sum_{m=L, R} \kappa_{f f^{\prime}}^{A} g_{f f^{\prime}}^{m} A_{1 / 2}^{A}\left(\tau_{f}, \lambda_{f^{\prime}}\right)\right)\left.\right|^{2},
\end{aligned}
$$

\footnotetext{
${ }^{2}$ The analytical expressions for $\phi \rightarrow g g$ decays are easily obtainable from those for $\phi \rightarrow \gamma \gamma$. Similarly, for $g g \rightarrow \phi$ production.
} 

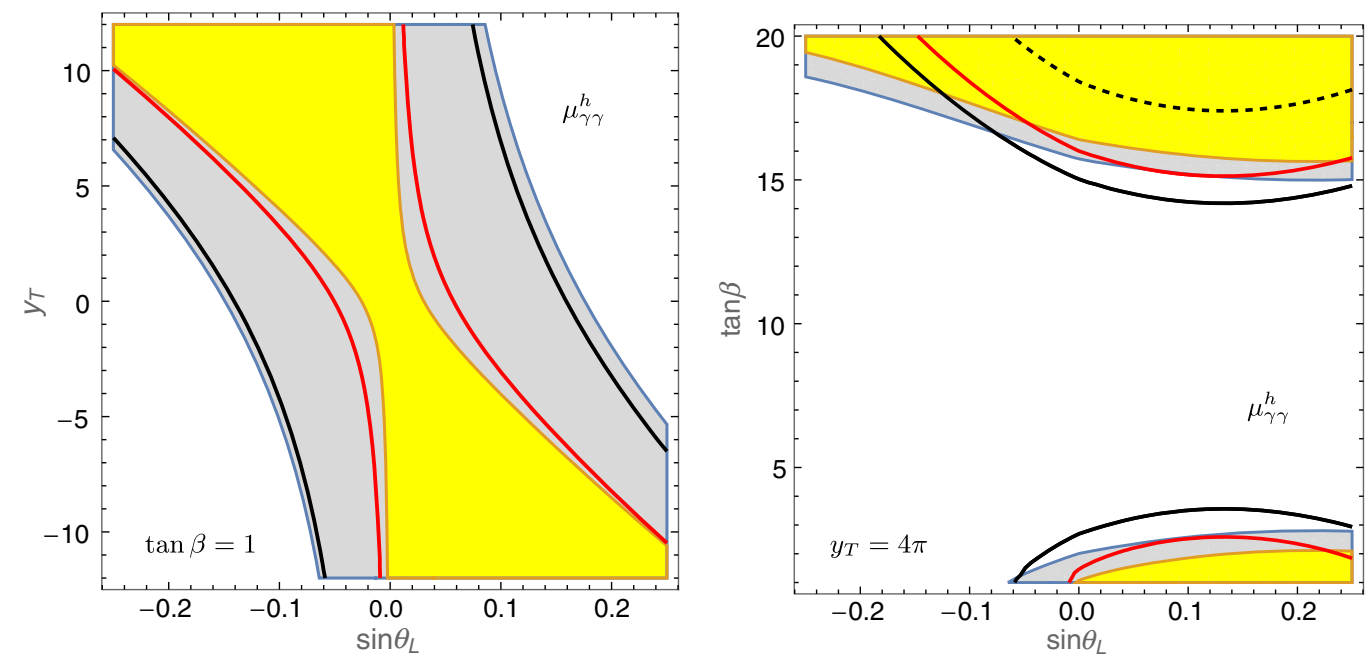

FIG. 4. Allowed regions for $\mu_{\gamma \gamma}^{h}$ in the $2 \mathrm{HDM}+\mathrm{VLQ}$ at $95 \%$ C.L. of Higgs data Run 1 (gray) and Run 2 (yellow) over the ( $s_{L}, y_{T}$ ) plane (left) with $\tan \beta=1$ and over the $\left(s_{L}, \tan \beta\right)$ plane (right) with $y_{T}=4 \pi$. The other parameters are fixed to $m_{T}=1 \mathrm{TeV}$, $\cos (\beta-\alpha)=0.05$. The contour plots correspond to $\mu_{Z \gamma}^{h}=1$ (dashed black), 1.5 (solid red), and 2 (solid black).

where $\phi=h\left(\equiv h_{\mathrm{SM}}\right)$ or $H$ are the $C P$-even Higgs bosons of the 2HDM, with $\kappa_{W W}^{h(H)}=\sin (\beta-\alpha)(\cos (\beta-\alpha))$. The relevant loop functions can be found in, e.g., Refs. [62,63]. Clearly, the charged Higgs boson contributions are smaller compared to the fermionic ones. Even for large $g_{\phi H^{ \pm} H^{\mp}}$, the charged Higgs effects are still negligible; henceforth, we neglect these.

The relevant modifications to the signal strength $\mu_{\gamma \gamma}^{h}$ (a function of the production cross sections and decay BRs) are defined in our scenario as

$$
\begin{aligned}
\mu_{V \gamma}^{h} & \equiv \frac{\sigma^{\mathrm{VLQ}}(p p \rightarrow \phi)}{\sigma\left(p p \rightarrow h_{\mathrm{SM}}\right)} \times \frac{\mathrm{BR}^{\mathrm{VLQ}}(\phi \rightarrow V \gamma)}{\operatorname{BR}\left(h_{\mathrm{SM}} \rightarrow V \gamma\right)}, \quad V=Z, \gamma, \\
\phi & =H, A \text { (summed over). }
\end{aligned}
$$

These come from the presence of an additional VLQ in the loops as well as from the modification of the $h t \bar{t}$ coupling for both Higgs production $(g g \rightarrow \phi)$ and Higgs decay $(\phi \rightarrow V \gamma)$. The theoretical value for $\mu_{\gamma \gamma}^{h}$ will depend on $m_{T}, s_{L}$ as well as the new Yukawa $y_{T}$. However, in the decoupling limit $\cos (\beta-\alpha)=0$, the dependence of $h t \bar{t}$ and $h T \bar{T}$ on $y_{T}$ cancels due to a factor $\cos (\beta-\alpha)=0$. What then remains is solely an $m_{T}$ and $s_{L}$ dependence, at least in the $V=\gamma$ case. The formula in Eq. (30) holds for the $Z \gamma$ case as well, wherein, however, the role of the $T$ loops can be altered significantly relative to that of the others by the additional degree of freedom carried by the $Z T \bar{T}$ vertex (unlike the case of the $\gamma T \bar{T}$ one, which is fixed by the Ward identity). Further, unlike the case of $\gamma \gamma, Z \gamma$ also benefits from nondiagonal loop transitions wherein the vertices $H$, $A t \bar{T}$, and $Z t \bar{T}$ (and c.c.) are involved. These differences between the two decay channels will play a key role in the remainder of our analysis.

The effects of a new heavy quark, $T$, have direct consequences for the signal strengths of the SM-like
Higgs boson. In Fig. 4, we illustrate a contour plot for $\mu_{\gamma \gamma}^{h}$ over the $\left(s_{L}, y_{T}\right)$ plane. The dashed black, solid black, and solid red contours capture $\mu_{Z_{\gamma}}^{h}=1,1.5$, and 2, respectively. The three contours fall within $1 \sigma$ of the ATLAS and CMS measured value of $\mu_{\gamma \gamma}^{h}=1.10 \pm$ 0.23 (stat) \pm 0.22 (syst). One can therefore conclude that this LHC constraint is less stringent than the oblique parameters previously discussed. However, once the $\mu_{\gamma \gamma}^{h}$ measurement improves with Run 2 data from the LHC and reaches the level of $10 \%$ or less deviation from the SM value, then the $\approx 125 \mathrm{GeV}$ diphoton event constraints will be more stringent. The pattern of $\mu_{Z_{\gamma}}^{h}$ is also given. It is remarkable that, for $\mu_{\gamma \gamma}^{h}$ compatible with LHC data at the $\pm 2 \sigma$ level, $\mu_{Z \gamma}^{h}$ can see an enhancement up to a factor of nearly 2 .

\section{CONFRONTING THE 2HDM + VLQ WITH LHC DATA}

In order to explain the LHC data in the framework of our $2 \mathrm{HDM}+\mathrm{VLQ}$ construct, we consider the $g g \rightarrow \phi$ and $\phi \rightarrow$ $\gamma \gamma$ (with $\phi=H$ and $A$ ) processes where the contribution of all the quarks including $T$ is considered. In the SM, only the top-quark loop gives a significant fermionic contribution. Besides the top quark, the new VLQ state $T$ can also contribute in the 2HDM + VLQ case, for both the SM-like and the other heavy Higgs production modes in addition to their decays into diphotons.

We start by assuming that we are in the alignment limit of the light Higgs boson $h, \cos (\beta-\alpha)=0$, wherein the heavier $C P$-even Higgs boson, $H$, decays to $W^{+} W^{-}$and $Z Z$ vanish at the tree level, which is consistent with current Higgs boson searches. (Needless to say, the $A$ state cannot directly couple to pairs of SM massive gauge bosons in the presence of $C P$ conservation.) We also assume that the 

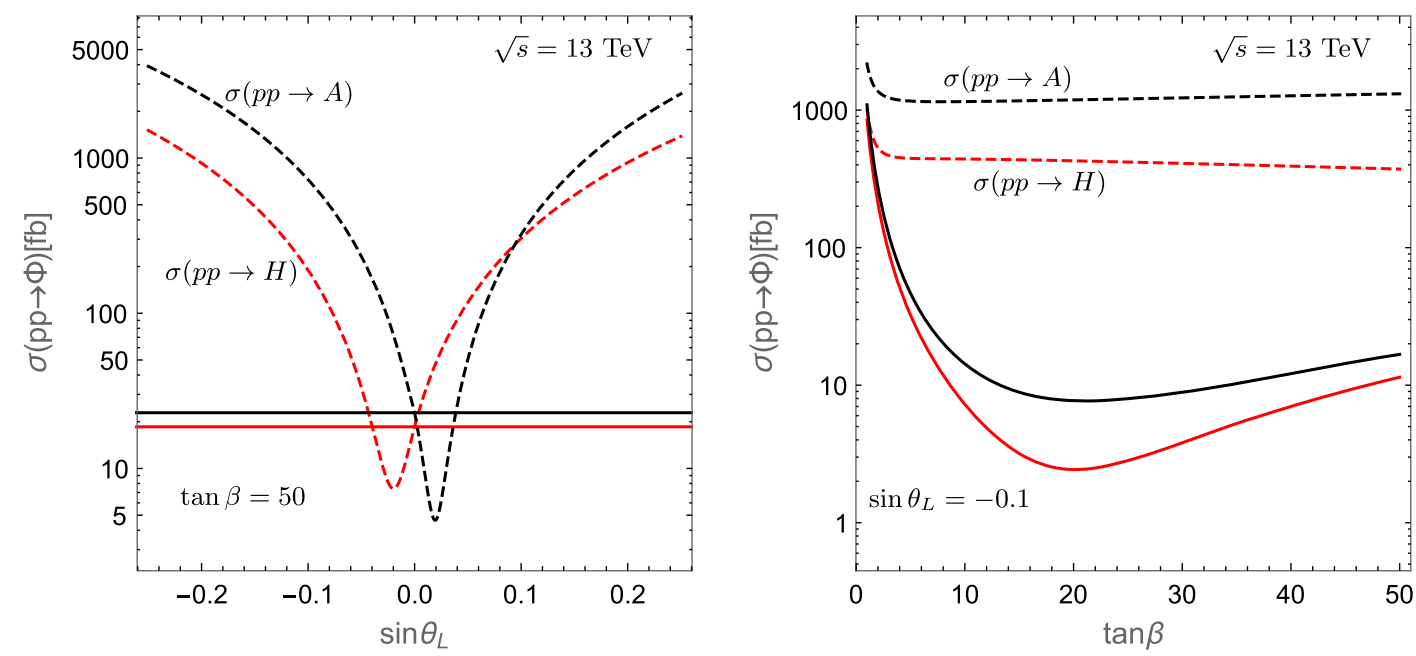

FIG. 5. Heavy Higgs production $\sigma(p p \rightarrow A, H)$ in fb at the LHC with $\sqrt{s}=13 \mathrm{TeV}$ as a function of $\sin \theta_{L}$ (left) and tan $\beta$ (right) for $m_{H}=m_{A}=700 \mathrm{GeV}$ in the alignment limit of the $2 \mathrm{HDM}+\mathrm{VLQ}$. We fix $y_{T}=2 \pi, m_{T}=700 \mathrm{GeV}$, and $\cos (\beta-\alpha)=0$. The solid (black and red) lines correspond to 2HDM without VLQ.

heavy Higgs states are degenerate, $m_{H}=m_{A}$, which is favored by satisfying theoretical bounds such as vacuum stability, perturbativity, and those allowed by EWPOs [64].

The cross section for this process is given by

$$
\begin{aligned}
\sigma^{\mathrm{VLQ}}(p p \rightarrow \phi) & =\sigma\left(p p \rightarrow h_{\mathrm{SM}}\right) \times \frac{\Gamma^{\mathrm{VLQ}}(\phi \rightarrow g g)}{\Gamma\left(h_{\mathrm{SM}} \rightarrow g g\right)}, \\
\phi & =H, A
\end{aligned}
$$

where $\Gamma^{\mathrm{VLQ}}(\phi \rightarrow g g)$ is the $g g$ width of the SM augmented by the extra VLQ loop contribution. The SM Higgs cross section is taken from the Higgs working group study of Ref. [65]. In our 2HDM + VLQ scenario, the cross section can be enhanced from the additional VLQ loop, which introduces the $h T \bar{T}$ coupling which can be large. Its sign is such that it can enable constructive interference with the top

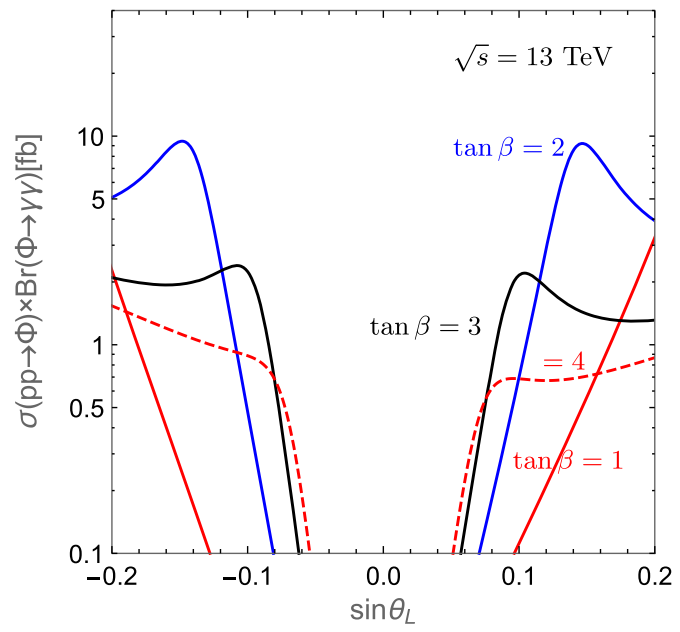

quark loop. Furthermore, the $\mathrm{BR}^{\mathrm{VLQ}}(H, A \rightarrow \gamma \gamma)$ can overall be enhanced as well through a similar dynamics, though it should be recalled here that the dominant loop is due to $W^{ \pm}$'s which typically have an opposite sign to the $t$ and $T$ loops, owing to the different spin statistics. In Fig. 5, fixing $m_{H}=m_{A}=700 \mathrm{GeV}$, we present the dependence of $\sigma(p p \rightarrow H, A)$ upon $s_{L}$ for $\tan \beta=50$ while the right panel shows the same quantity as a function of $\tan \beta$ with fixed mixing angle $s_{L}=-0.1$. Both panels are for a large Yukawa of the new top, $y_{T}=2 \pi$ with $m_{T}=700 \mathrm{GeV}$. Here, $\tan \beta$ is required to be smaller than 20 for $m_{H}=m_{A}=700 \mathrm{GeV}$. It is clear from Fig. 5 that, away from the $s_{L} \approx 0$ limit (left frame), the $p p \rightarrow H, A$ process can differ by 2 orders of magnitude compared to the ordinary $2 \mathrm{HDM}$-II.

In the left panel of Fig. 6 we present the dependence of $\sigma(p p \rightarrow \phi) \times \operatorname{BR}(\phi \rightarrow \gamma \gamma)$ upon the mixing angle $s_{L}$ for

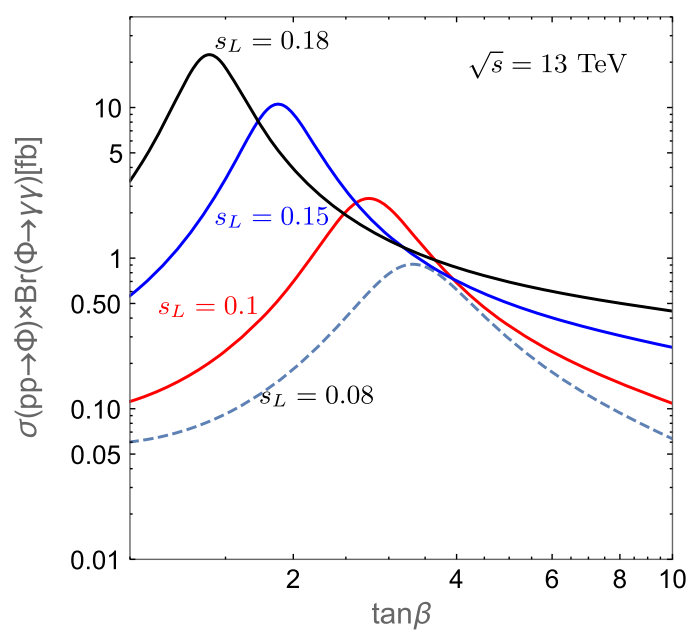

FIG. 6. Cross sections of $(p p \rightarrow H, A \rightarrow \gamma \gamma)$ in fb in the 2HDM + VLQ as a function of $s_{L}$ for different values of tan $\beta$ (left) and tan $\beta$ for different values of $s_{L}$ (right). Here, $\cos (\beta-\alpha)=0, m_{H}=m_{A}=700 \mathrm{GeV}, m_{T}=700 \mathrm{GeV}$, and $y_{T}=4 \pi$. 
$\tan \beta=1,2,3,4$ while the right panel shows the same quantity as a function of $\tan \beta$ with fixed mixing angles $s_{L}$. Both panels are for a large Yukawa of the new top, $y_{T}=4 \pi$. In this illustration, we limit ourselves to low $\tan \beta$ values which are favored by $\tau \tau$ LHC data and also because of perturbative unitarity coming from the 2HDM scalar potential. We emphasize that, in the decoupling limit which we consider, the $W^{ \pm}$loop in $H \rightarrow \gamma \gamma$ vanishes since it is proportional to $\cos (\beta-\alpha) \approx 0$ while $A \rightarrow \gamma \gamma$ has no $W^{ \pm}$loop at all because of the $C P$-odd nature of the $A$ state. Furthermore, for $H, A \rightarrow g g$, we are only left with top quarks and VLQ contributions. It is clear from Fig. 6 that, away from the $s_{L} \approx 0$ limit (left frame), the $p p \rightarrow H$, $A \rightarrow \gamma \gamma$ process can significantly contribute to the high mass diphoton event sample. Hence, the recent studies carried out by ATLAS and CMS have the potential to significantly constrain our model. For example, for $H, A$ masses around $500 \mathrm{GeV}, \tan \beta$ values of $2-3$ are not possible, as no particular feature has emerged from the LHC data in the relevant $m_{\gamma \gamma}$ invariant mass range (hence they are compatible with the SM rates, driven by $q \bar{q}, g g \rightarrow \gamma \gamma$ events). In fact, the strongest constraints would emerge (right frame) for any $m_{A}=m_{H}$ value whenever a loop threshold opens up, whether this is the $t \bar{t}$ one at $350 \mathrm{GeV}$ or the $T \bar{T}$ one at higher energies (possibly excluding $\tan \beta \approx 4$ values), which may happen through both Higgs [66] and $Z$ [67,68] boson mediation.

A point we have previously made regarding our $2 \mathrm{HDM}+\mathrm{VLQ}$ construct is the possibility of $\gamma \gamma$ rates at high invariant masses being compatible with Run 2 data with the $Z \gamma$ ones being potentially different from the standard 2HDM-II case. With this in mind, we present Fig. 7, where the inclusive rates of these two channels in the $2 \mathrm{HDM}+\mathrm{VLQ}$ are shown, divided by the corresponding
$2 \mathrm{HDM}$ rates (these correspond to the case of $s_{L}=0$ and $\left.m_{T} \rightarrow \infty\right)$, i.e.,

$\mu_{p p}^{V \gamma} \equiv \frac{\sigma(g g \rightarrow H, A \rightarrow V \gamma)_{2 \mathrm{HDM}+\mathrm{VLQ}}}{\sigma(g g \rightarrow H, A \rightarrow V \gamma)_{2 \mathrm{HDM}}}, \quad V=Z, \gamma$,

for low $\tan \beta$ and negative $y_{T}$. It is clear that substantial differences (up to a factor of 2) can exist between the two scenarios, so long that $s_{L}$ is sizably different from zero. (Local maxima in the plot correspond to relative sign changes between the VLQ loop contributions and the $2 \mathrm{HDM}$ ones.) In fact, over most of the possible $s_{L}$ interval, both $\gamma \gamma$ and $Z \gamma$ in the 2HDM + VLQ depart simultaneously from the ordinary $2 \mathrm{HDM}-\mathrm{II}$ case.

As pointed out in the Introduction, ATLAS and CMS have reported a possible increase in the signal strength of the $t \bar{t} h$ associated production mode in the LHC data. The most recent preliminary results from Run 2 relayed by CMS still show an enhancement of $\mu_{t \overline{t h}}^{p p}=1.5 \pm 0.5$ times the SM prediction with an observed significance of $3.3 \sigma$ compared to the expected one of $2.5 \sigma$ (obtained from combining results of Run 1). Many different final states contribute to this enhancement, but the most significant excesses are observed in multilepton final states which probe closely $t \bar{t} h$ production. One possibility to explain the excess is that it could be due to the modified Higgs coupling to the SM top quark, resulting in an enhanced $t \bar{t} h$ production. Mixing within the top sector, i.e., between the $t$ and $T$ states, also allows for a sufficiently large enhancement of the $p p \rightarrow t \bar{t} h$ rates. Hence, a possibility offered by our scenario could be the potential to explain a $t \bar{t} h$ enhancement. In this case, rates for the loop-induced processes of the $h$ should remain SM-like, despite the VLQ contributions in our scenario.
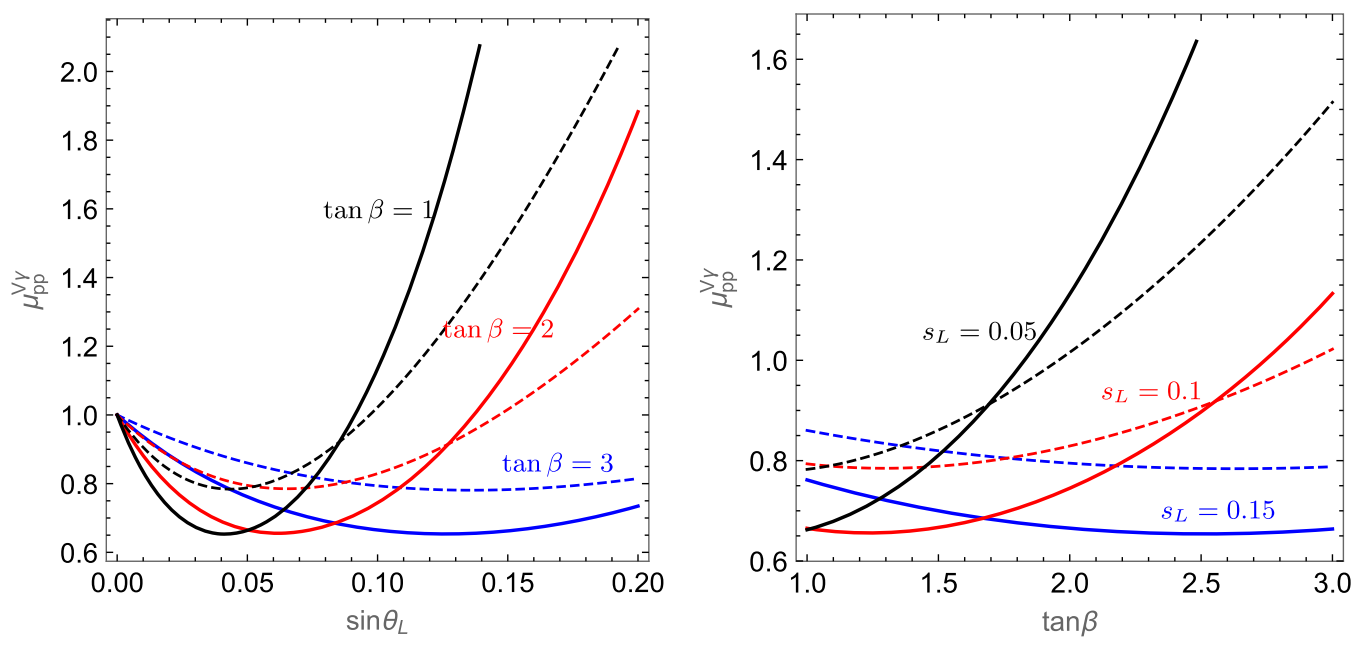

FIG. 7. Ratios of $\sigma(g g \rightarrow H, A \rightarrow \gamma \gamma)$ (solid lines) and $\sigma(g g \rightarrow H, A \rightarrow Z \gamma)$ (dashed lines) in the 2HDM + VLQ over the 2HDM for $m_{\gamma \gamma}=m_{Z \gamma}=750 \mathrm{GeV}$ as a function of $s_{L}$ for different values of $\tan \beta$ (left) and Yukawa coupling $y_{T}$ (right). Here, $\cos (\beta-\alpha)=0$, $m_{H}=m_{A}=700 \mathrm{GeV}, m_{T}=700 \mathrm{GeV}$. Also, we fix $y_{T}=-2 \pi$. 

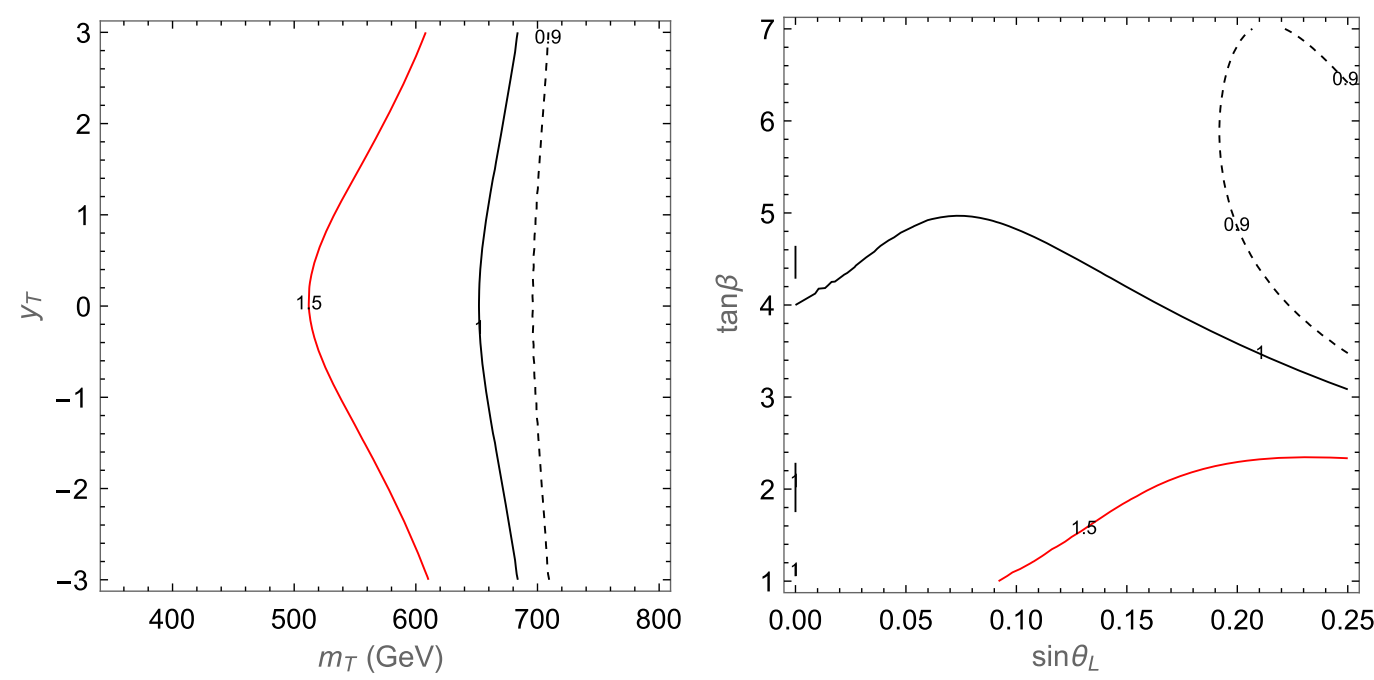

FIG. 8. Contour plots for the Higgs signal strength $\mu_{t \bar{t} h}^{p p}$ over the $\left(\sin \theta_{L}, \tan \beta\right)$ plane (right) and over the $\left(m_{T}, y_{T}\right)$ plane (left) in the $2 \mathrm{HDM}+\mathrm{VLQ}$ with $\cos (\beta-\alpha)=0, m_{h}=125.5 \mathrm{GeV}, m_{H}=m_{A}=m_{H^{ \pm}}=600 \mathrm{GeV}$ for $\left(\sin \theta_{L}, \tan \beta\right)=(0.22,1.5)$ in the left panel and $\left(m_{T}, y_{T}\right)=(400 \mathrm{GeV}, 2)$ in the right panel, at the LHC Run 2. In both plots the red line corresponds to $\mu_{t \bar{t} h}^{p p}=1.5$, the solid black one to $\mu_{t \bar{h} h}^{p p}=1$, and the dashed black one to $\mu_{t \bar{t} h}^{p p}=0.9$.

In Fig. 8 we show contour plots of $\mu_{t \bar{p}}^{p p}$ in the $\left(\sin \theta_{L}\right.$, $\tan \beta)$ plane (right) and $\left(m_{T}, y_{T}\right)$ plane (left) in the $2 \mathrm{HDM}+$ VLQ given SM-like Higgs couplings. As can be seen from the figure, there is a strong dependence upon both the parameters $\sin \theta_{L}$ and $\tan \beta$. Clearly, the $2 \mathrm{HDM}+\mathrm{VLQ}$ can reproduce a higher value of the $t \bar{t} h$ signal strength than in the $\mathrm{SM}$, typically $\mu_{t \bar{t} h}^{p p} \approx 1.5$, for small $\tan \beta$ and $\sin \theta_{L}=0.22$, i.e., a parameter space configuration ideally testable within the experimental range of LHC Run 2 through direct $T$ production.

In fact, in the light of a possible explanation of potentially anomalous $t \bar{t} h$ data afforded by a heavy top with $m_{T} \approx 600 \mathrm{GeV}$, we end this section with a few comments on the possible production and decay patterns for such a VLQ state, as the ensuing signatures would be a distinctive feature between a standard 2HDM-II and its VLQ version. Unlike the case of the SM + VLQ framework where the BR of $T \rightarrow b W^{+}, T \rightarrow t Z$, and $T \rightarrow t h$ are, respectively, $50 \%, 25 \%$, and $25 \%$ for heavy $m_{T}$, in models with more than one Higgs doublet, several decay patterns can appear from the interaction of the new heavy quark with the extended Higgs sector, e.g.,

$$
T \rightarrow b W^{+}, \quad t Z, \quad t h, \quad t H, \quad t A, \quad b H^{+},
$$

where the last three cases are unique to a $2 \mathrm{HDM}$ sector. (The partial widths for all these modes are given in Appendix C.) In Fig. 9 we illustrate the BRs of the $T$

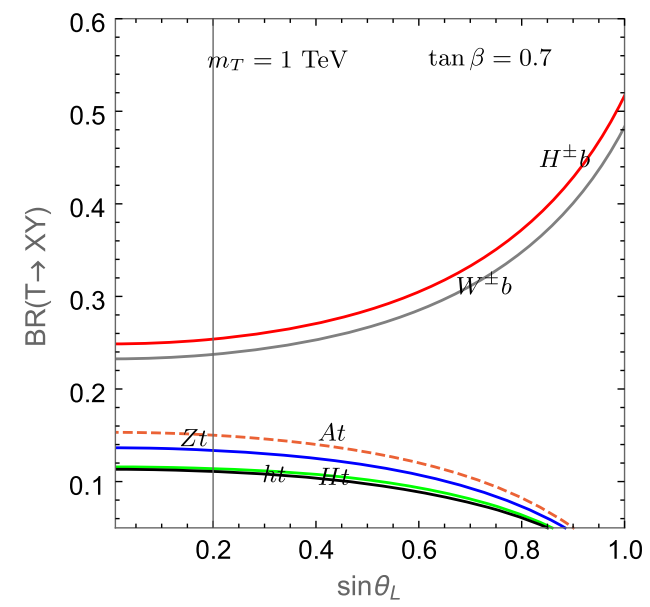

FIG. 9. Branching ratios of $T$ in the $2 \mathrm{HDM}+$ VLQ as a function of $\tan \beta$ (left) and a a function of $\sin \theta_{L}$ (right) with $y_{T}=2$, $m_{h}=125.5 \mathrm{GeV}, m_{A}=m_{H}=500 \mathrm{GeV}, m_{H^{ \pm}}=600 \mathrm{GeV}, \cos (\beta-\alpha)=0, m_{T}=1 \mathrm{TeV}$ with $\tan \beta=0.7$ (right) and $\sin \theta_{L}=V_{T b}=$ $10^{-2}$ (left). 

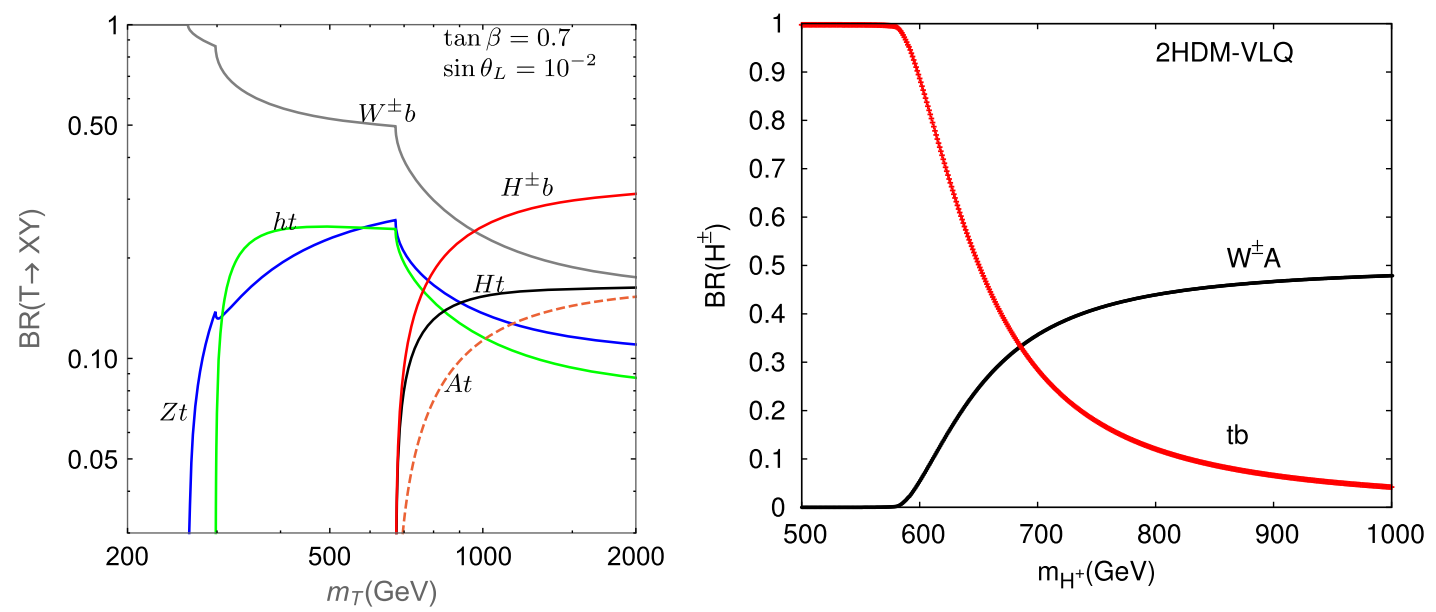

FIG. 10. Left: BRs of the $T$ as a function of $m_{T}$ with the same parameter as in Fig. 9. Right: BRs of the $H^{ \pm}$state in the $2 \mathrm{HDM}+\mathrm{VLQ}$ as a function of its mass for $\sin \theta_{L}=0, y_{T}=6, m_{h}=125.5 \mathrm{GeV}, m_{A}=m_{H}=500 \mathrm{GeV}, \cos (\beta-\alpha)=0, \tan \beta=0.7$, and $m_{T}=1000 \mathrm{GeV}$. (Here, we assume $m_{T}>m_{H^{ \pm}}$, so that $2 \mathrm{HDM}$-like decays only are included; i.e., the $H^{ \pm}$state cannot decay into final states with $T$ 's.)

quark as a function of $\sin \theta_{L}$ (right panel) and as a function of $\tan \beta$ (left panel). We assume a heavy scalar scenario where $m_{H}=m_{A}=500 \mathrm{GeV}, H^{ \pm}=600 \mathrm{GeV}$, and $m_{T}=$ $1 \mathrm{TeV}$. As seen from the right plot for $\tan \beta=0.7$, $T \rightarrow b W^{+}$, and $T \rightarrow b H^{+}$are comparable and could reach $25 \%$ at small mixing $\sin \theta_{L}=0$ instead of $50 \%$ in the SM for $T \rightarrow b W^{+}$. However, $T \rightarrow t h$ and $t Z$ are slightly smaller compared to the SM + VLQ case (less than 12\%), a function of $\tan \beta$ and with small mixing $\sin \theta_{L}=10^{-2}$. We see that in the limit $\sin \theta_{L} \rightarrow 0$, when the nonstandard $T$ decay modes $T \rightarrow t H, t A$ are suppressed due to their coupling, which is proportional to $\sin \theta_{L}, T \rightarrow b H^{+}$is comparable to $T \rightarrow b W^{+}$for $\tan \beta \approx 0.5-0.7$ and may offer an alternative discovery mode. We stress here that the $b \rightarrow s \gamma$ constraint is fulfilled for such small $\tan \beta \approx 0.5-0.7$ if $m_{H^{ \pm}}=600 \mathrm{GeV}$.

Finally, in Fig. 10 (left) we illustrate the branching fractions of the heavy top as a function of $m_{T}$. As it can be seen, when non-SM decays such as $T \rightarrow b H^{+}, t H, t A$ are not open, the situation is similar to the SM + VLQ case, as expected. However, when $T \rightarrow b H^{+}, t H, t A$ are open, one can see that $T \rightarrow b H^{+}$compete with $T \rightarrow b W^{+}$and even dominate for high $m_{T}$. Note that, at large $m_{T}, T \rightarrow t H, t A$ are slightly larger than the SM decays $T \rightarrow t h, t Z$.

There exist several LHC analyses searching for (modelindependent) pair produced new VLQ states, performed by both ATLAS and CMS. These place limits in the range $600-800 \mathrm{GeV}$ depending on the actual BR of the $T$ quark in the channels searched for. These do not presently include the $T \rightarrow b H^{+}$mode. However, with $m_{T}=1000 \mathrm{GeV}$, the $T \bar{T} \rightarrow b \bar{b} H^{+} H^{-}$mode (followed by $H^{+} H^{-} \rightarrow b \bar{b} b \bar{b} W^{+} W^{-}$ decays) can lead to " $W^{+} W^{-}$plus $6 b$-jets" as a sizable and (very distinctive) signature. Even with small $\sin \theta_{L}$, an interesting possibility would be $T \bar{T} \rightarrow b \bar{b} W^{+} H^{-}$ decays, with $H^{-} \rightarrow b \bar{b} W^{-}$, producing an equally distinctive
" $W^{+} W^{-}$plus $4 b$-jets" signal. While the " $W^{+*} W^{-*} W^{+} W^{-}$ plus $4 b$-jets" case is also a potentially interesting channel, stemming from $T \bar{T} \rightarrow t \bar{t} h h \rightarrow b \bar{b} W^{+} W^{-} h h$ with $h h \rightarrow$ $b \bar{b} W^{+*} W^{-*}$ decays, given that $T \rightarrow t h$ is probably very difficult to detect (as intimated already; see [69]), the alternative of accessing the new VLQ state via $T \rightarrow b H^{+}$ decays becomes a very intriguing one. In fact, this is the ideal channel to characterize our model, as even neutral $A$, $H$ decays are, so to say, "degenerate" (i.e., they can have the same decay patterns) with those from the SM-like Higgs state, $h$. Needless to say, to establish this signature would represent circumstantial evidence of a $2 \mathrm{HDM}+\mathrm{VLQ}$ structure. Typical $H^{ \pm}$decay patterns can be found in Fig. 10 (right), showing that $b \bar{b} W^{ \pm}$decays of charged Higgs bosons (via $t b, W^{ \pm} A$ ) are indeed the dominant ones for large $m_{H^{ \pm}}$values.

\section{CONCLUSIONS}

In this paper, we have extended the ordinary 2HDM-II by a singlet heavy VLQ with the same EM charge as the top quark. In the (near) decoupling limit of the 2HDM-II, one neutral $C P$-even Higgs state, $h$, can mimic the SM-like Higgs boson seen at the LHC Run 1 at $\approx 125 \mathrm{GeV}$ while the other two neutral Higgs states, $H$ and $A$, can be constrained as the model has to accommodate the data observed by ATLAS and CMS at the LHC Run 2 in both the low and the high mass regions, which are to date consistent with SM expectations. We have then proceeded to a phenomenological comparison between the 2HDM + VLQ scenario and the ordinary $2 \mathrm{HDM}$ case, limitedly to the LHC environment.

We have illustrated that a different decay pattern emerges in the $2 \mathrm{HDM}+\mathrm{VLQ}$ with respect to the standard 2HDM when $\gamma \gamma$ and $Z \gamma$ samples are compared to each other. 
Then, we have shown that, at the same time, an enhancement of $\sigma(p p \rightarrow t \bar{t} h)$ by a factor of up to 2 can occur, which would explain an increased value of such a cross section at the LHC, i.e., in the direction of a possible enhancement seen in Run 2 data. All this can occur for VLQ masses of order $600-800 \mathrm{GeV}$, so that we have finally highlighted that non-SM-like decays of this VLQ state, particularly via $H^{ \pm}$channels, would be evidence of a 2HDM sector.

In fact, by combining these instances, a peculiar "smoking-gun" situation may emerge in the $2 \mathrm{HDM}+\mathrm{VLQ}$ scenario discussed here, where one has $\gamma \gamma$ rates at large invariant masses essentially compatible with the SM background, yet a depletion (with respect to the 2HDM yield) can be seen in the $Z \gamma$ sample, with or without an enhancement of the $t \bar{t} h$ cross section. One could indeed disentangle this as being due to this particular BSM structure by finally revealing a variety of $T \rightarrow b H^{+}$decays emerging from QCD induced $T \bar{T}$ production.

Remarkably, all such phenomenology can be obtained for parameter space configurations compliant with current theoretical and experimental constraints, as we have scrupulously assessed using up-to-date tools, yet amenable to prompt phenomenological investigation in the upcoming years at the LHC, during Runs 2 and 3.

\section{ACKNOWLEDGMENTS}

The authors are supported by Grant H2020-MSCARISE-2014 No. 645722 (NonMinimalHiggs). This work is also supported by the Moroccan Ministry of Higher Education and Scientific Research MESRSFC and CNRST: Project PPR/2015/6. S. M. is supported in part through the NExT Institute. R. B. and C. S. U. thank S. M. for hospitality in Southampton when part of this research was carried out. A. A. and R. B. would also like to acknowledge the hospitality of the National Center for Theoretical Sciences (NCTS), Physics Division, in Taiwan.

\section{APPENDIX A: YUKAWA COUPLINGS IN THE 2HDM + VLQ}

These are as follows:

$$
\begin{gathered}
\kappa_{t \bar{t}}^{h}=c_{L} c_{R}\left(y_{t}^{h}\right)-s_{R} c_{L}\left(c_{\beta \alpha} \frac{y_{T}}{y_{t}}+s_{\beta \alpha} \frac{\xi_{T}}{y_{t}}\right), \\
\kappa_{T \bar{T}}^{h}=s_{L} s_{R}\left(y_{t}^{h}\right)+s_{L} c_{R}\left(c_{\beta \alpha} \frac{y_{T}}{y_{t}}+s_{\beta \alpha} \frac{\xi_{T}}{y_{t}}\right), \\
\kappa_{t \bar{T}}^{h}=c_{R} s_{L}\left(y_{t}^{h}\right)-s_{R} s_{L}\left(c_{\beta \alpha} \frac{y_{T}}{y_{t}}+s_{\beta \alpha} \frac{\xi_{T}}{y_{t}}\right),
\end{gathered}
$$

$$
\begin{gathered}
\kappa_{t \bar{t}}^{H}=c_{L} c_{R}\left(y_{t}^{H}\right)+s_{R} c_{L}\left(s_{\beta \alpha} \frac{y_{T}}{y_{t}}-c_{\beta \alpha} \frac{\xi_{T}}{y_{t}}\right) \\
\kappa_{T \bar{T}}^{H}=s_{L} s_{R}\left(y_{t}^{H}\right)-s_{L} c_{R}\left(s_{\beta \alpha} \frac{y_{T}}{y_{t}}-c_{\beta \alpha} \frac{\xi_{T}}{y_{t}}\right) \\
\kappa_{t \bar{T}}^{H}=c_{R} s_{L}\left(y_{t}^{H}\right)+s_{R} s_{L}\left(s_{\beta \alpha} \frac{y_{T}}{y_{t}}-c_{\beta \alpha} \frac{\xi_{T}}{y_{t}}\right) \\
\kappa_{t \bar{t}}^{A}=i\left(c_{L} c_{R}\left(y_{t}^{A}\right)+s_{R} c_{L} \frac{y_{T}}{y_{t}}\right) \\
\kappa_{T \bar{T}}^{A}=i\left(s_{L} s_{R}\left(y_{t}^{A}\right)-s_{L} c_{R} \frac{y_{T}}{y_{t}}\right) \\
\kappa_{t \bar{T}}^{A}=i\left(s_{L} c_{R}\left(y_{t}^{A}\right)+s_{R} s_{L} \frac{y_{T}}{y_{t}}\right)
\end{gathered}
$$

The above reduced Higgs couplings $\kappa_{i j}^{\phi}$ are expressed in terms of the normalized $y_{t}^{\phi}$ ones given by

$$
\begin{aligned}
y_{t}^{h} & =\sin (\beta-\alpha)+\cot \beta \cos (\beta-\alpha), \\
y_{t}^{H} & =\cos (\beta-\alpha)-\cot \beta \sin (\beta-\alpha), \\
y_{t}^{A} & =\cot \beta .
\end{aligned}
$$

It is easy to check that, in the case of zero mixing $s_{L}=0$, the $\{h, H, A\} t \bar{t}$ couplings reduce to the $2 \mathrm{HDM}$ ones while $\{h, H, A\} t \bar{T}$ and $\{h, H, A\} T \bar{T}$ all vanish.

\section{APPENDIX B: FORM FACTORS FOR $b \rightarrow s \gamma$}

These were used as follows:

$$
\begin{aligned}
& f_{1 \gamma}(x)=\frac{x}{72}\left[\frac{8 x^{2}+5 x-7}{(1-x)^{3}}-\frac{6 x(2-3 x)}{(1-x)^{4}} \ln (x)\right], \\
& f_{1 G}(x)=\frac{x}{24}\left[\frac{x^{2}-5 x-2}{(1-x)^{3}}-\frac{6 x}{(1-x)^{4}} \ln (x)\right], \\
& f_{2 \gamma}(x)=\frac{x}{12}\left[\frac{3-5 x}{(1-x)^{2}}+\frac{2(2-3 x)}{(1-x)^{3}} \ln (x)\right] \\
& f_{2 G}(x)=\frac{x}{4}\left[\frac{3-x}{(1-x)^{2}}+\frac{2}{(1-x)^{3}} \ln (x)\right]
\end{aligned}
$$

\section{APPENDIX C: PARTIAL WIDTHS OF THE VLQ}

In this appendix we give the analytic expressions of the partial widths of the VLQ into vector and Higgs bosons, $T \rightarrow q V$ and $T \rightarrow q \phi$, as

$$
\Gamma(T \rightarrow q V)=\frac{g^{2}}{32 \pi} \frac{\beta}{m_{T}^{2}}\left(\left(g_{L}^{2}+g_{R}^{2}\right)\left(\frac{m_{T}^{2}+m_{q}^{2}}{2}+\frac{\left(m_{T}^{2}-m_{q}^{2}\right)^{2}}{2 m_{V}^{2}}-m_{V}^{2}\right)-6 g_{L} g_{R} m_{T} m_{q}\right),
$$




$$
\Gamma(T \rightarrow q \phi)=\frac{\beta}{8 \pi m_{T}^{2}}\left(\left(g_{L}^{2}+g_{R}^{2}\right) \frac{m_{T}^{2}+m_{q}^{2}-m_{\phi}^{2}}{2}+g_{L} g_{R} m_{T} m_{q}\right),
$$

with

$$
\beta=\frac{\left(\left(m_{T}^{2}-\left(m_{q}+m_{X}\right)^{2}\right)\left(m_{T}^{2}-\left(m_{q}-m_{X}\right)^{2}\right)\right)^{p}}{2 m_{T}} \quad(X=V, \phi),
$$

where $m_{V}\left(m_{\phi}\right)$ and $m_{q}$ are the masses of the gauge(Higgs) bosons and the SM quark, respectively. We denote as $g_{L}$ and $g_{R}$ the left- and right-handed components of the SM quark $q$. Finally, $p=1 / 2$ for $\phi=h, H$ and $p=3 / 2$ for $\phi=A$.

[1] G. Aad et al. (ATLAS Collaboration), Phys. Lett. B 716, 1 (2012); S. Chatrchyan et al. (CMS Collaboration), Phys. Lett. B 716, 30 (2012).

[2] S. Chatrchyan et al. (CMS Collaboration), Phys. Lett. B 716, 30 (2012).

[3] https://twiki.cern.ch/twiki/bin/view/AtlasPublic.

[4] https://twiki.cern.ch/twiki/bin/view/AtlasPublic/Winter2016$13 \mathrm{TeV}$.

[5] https://twiki.cern.ch/twiki/bin/view/CMSPublic/Physics ResultsHIG.

[6] N. Arkani-Hamed, A. G. Cohen, E. Katz, A. E. Nelson, T. Gregoire, and J. G. Wacker, J. High Energy Phys. 08 (2002) 021.

[7] H. C. Cheng and I. Low, J. High Energy Phys. 09 (2003) 051.

[8] H. C. Cheng and I. Low, J. High Energy Phys. 08 (2004) 061.

[9] I. Low, J. High Energy Phys. 10 (2004) 067.

[10] J. Hubisz and P. Meade, Phys. Rev. D 71, 035016 (2005).

[11] H. C. Cheng, I. Low, and L.T. Wang, Phys. Rev. D 74, 055001 (2006).

[12] J. Hubisz, P. Meade, A. Noble, and M. Perelstein, J. High Energy Phys. 01 (2006) 135.

[13] I. Antoniadis, Phys. Lett. B 246, 377 (1990).

[14] T. Appelquist, H. C. Cheng, and B. A. Dobrescu, Phys. Rev. D 64, 035002 (2001).

[15] G. Servant and T. M. P. Tait, Nucl. Phys. B650, 391 (2003).

[16] C. Csaki, C. Grojean, J. Hubisz, Y. Shirman, and J. Terning, Phys. Rev. D 70, 015012 (2004).

[17] G. Cacciapaglia, A. Deandrea, and J. Llodra-Perez, J. High Energy Phys. 03 (2010) 083.

[18] T. Aaltonen et al. (CDF Collaboration), Phys. Rev. Lett. 106, 191801 (2011).

[19] T. Aaltonen et al. (CDF Collaboration), Phys. Rev. Lett. 107, 191803 (2011).

[20] G. Aad et al. (ATLAS Collaboration), Report No. ATLASCONF-2011-036.

[21] S. Chatrchyan et al. (CMS Collaboration), Report No. CMS-PAS-SUS-12-009.

[22] D. Barducci and L. Panizzi, J. High Energy Phys. 12 (2017) 057.
[23] G. C. Branco, P. M. Ferreira, L. Lavoura, M. N. Rebelo, M. Sher, and J. P. Silva, Phys. Rep. 516, 1 (2012).

[24] R. Benbrik, C. H. Chen, and T. Nomura, Phys. Rev. D 93, 055034 (2016).

[25] J. A. Aguilar-Saavedra, R. Benbrik, S. Heinemeyer, and M. Prez-Victoria, Phys. Rev. D 88, 094010 (2013).

[26] M. Badziak, Phys. Lett. B 759, 464 (2016).

[27] A. Angelescu, A. Djouadi, and G. Moreau, Phys. Lett. B 756, 126 (2016).

[28] M. Carena, I. Low, N. R. Shah, and C.E.M. Wagner, J. High Energy Phys. 04 (2014) 015.

[29] J. F. Gunion, H. E. Haber, G. L. Kane, and S. Dawson, Front. Phys. 80, 1 (2000).

[30] S. A. R. Ellis, R. M. Godbole, S. Gopalakrishna, and J. D. Wells, J. High Energy Phys. 09 (2014) 130.

[31] M. Misiak and M. Steinhauser, Eur. Phys. J. C 77, 201 (2017).

[32] G. Aad et al. (ATLAS and CMS Collaborations), J. High Energy Phys. 08 (2016) 045.

[33] P. Bechtle, O. Brein, S. Heinemeyer, O. Stål, T. Stefaniak, G. Weiglein, and K. E. Williams, Eur. Phys. J. C 74, 2693 (2014); Proc. Sci., CHARGED2012 (2012) 024.

[34] ATLAS Collaboration, Report No. ATLAS CONF-2016081.

[35] ATLAS Collaboration, Report No. ATLAS CONF-2016063.

[36] ATLAS Collaboration, Report No. ATLAS CONF-2016003.

[37] CMS Collaboration, Report No. CMS-PAS-HIG-16-020.

[38] CMS Collaboration, Report No. CMS-PAS-HIG-16-038.

[39] CMS Collaboration, Report No. CMS-PAS-HIG-16-020.

[40] D. Barducci, A. Belyaev, M. Buchkremer, J. Marrouche, S. Moretti, and L. Panizzi, Comput. Phys. Commun. 197, 263 (2015).

[41] D. Barducci, A. Belyaev, M. Buchkremer, G. Cacciapaglia, A. Deandrea, S. De Curtis, J. Marrouche, S. Moretti, and L. Panizzi, J. High Energy Phys. 12 (2014) 080.

[42] M. J. Dolan, J. L. Hewett, M. Krämer, and T. G. Rizzo, J. High Energy Phys. 07 (2016) 039.

[43] G. Aad et al. (ATLAS Collaboration), Report No. ATLASCONF-2013-018. 
[44] G. Aad et al. (ATLAS Collaboration), Phys. Rev. D 86, 012007 (2012).

[45] M. E. Peskin and T. Takeuchi, Phys. Rev. D 46, 381 (1992).

[46] L. Lavoura and J. P. Silva, Phys. Rev. D 47, 2046 (1993).

[47] T. Hahn, Comput. Phys. Commun. 140, 418 (2001).

[48] T. Hahn and M. Perez-Victoria, Comput. Phys. Commun. 118, 153 (1999).

[49] T. Hahn and M. Rauch, Nucl. Phys. B, Proc. Suppl. 157, 236 (2006).

[50] G. J. van Oldenborgh, Comput. Phys. Commun. 66, 1 (1991).

[51] T. Hahn, Proc. Sci., ACAT2010 (2010) 078.

[52] S. Dawson and E. Furlan, Phys. Rev. D 86, 015021 (2012).

[53] J. Beringer et al. (Particle Data Group), Phys. Rev. D 86, 010001 (2012).

[54] Y. Amhis et al., Eur. Phys. J. C 77, 895 (2017).

[55] M. Czakon, P. Fiedler, T. Huber, M. Misiak, T. Schutzmeier, and M. Steinhauser, J. High Energy Phys. 04 (2015) 168.

[56] M. Misiak et al., Phys. Rev. Lett. 114, 221801 (2015).

[57] F. Borzumati and C. Greub, Phys. Rev. D 58, 074004 (1998).
[58] M. Ciuchini, G. Degrassi, P. Gambino, and G. F. Giudice, Nucl. Phys. B527, 21 (1998).

[59] F. Borzumati and C. Greub, Phys. Rev. D 59, 057501 (1999).

[60] T. Hermann, M. Misiak, and M. Steinhauser, J. High Energy Phys. 11 (2012) 036.

[61] M. Blanke, A. J. Buras, K. Gemmler, and T. Heidsieck, J. High Energy Phys. 03 (2012) 024.

[62] M. Spira, Fortschr. Phys. 46, 203 (1998).

[63] A. Djouadi, Phys. Rep. 457, 1 (2008).

[64] K. A. Olive, Chin. Phys. C 40, 100001 (2016).

[65] https://twiki.cern.ch/twiki/bin/view/LHCPhysics/CERN YellowReportPageAt1314TeV.

[66] J. F. Gunion, H. E. Haber, G. Kane, and S. Dawson, The Higgs Hunter's Guide (Westview Press, Boulder, CO, 1990).

[67] S. Moretti, Phys. Rev. D 91, 014012 (2015).

[68] S. Jain, F. Margaroli, S. Moretti, and L. Panizzi, Phys. Rev. D 95, 014037 (2017).

[69] K. Harigaya, S. Matsumoto, M. M. Nojiri, and K. Tobioka, Phys. Rev. D 86, 015005 (2012). 